\title{
Discovery of a SARS-CoV-2 Broadly-Acting Neutralizing Antibody with Activity against Omicron and Omicron + R346K Variants
}

1 J. Andrew Duty ${ }^{2,3^{*}}$, Thomas Kraus ${ }^{2,3^{*}}$, Heyue Zhou ${ }^{1^{*}}$, Yanliang Zhang ${ }^{1^{*}}$, Namir Shaabani ${ }^{1}$, Soner Yildiz ${ }^{2,5}$,

$2 \mathrm{Na} \mathrm{Du}^{1}$, Alok Singh ${ }^{1}$, Lisa Miorin ${ }^{2,5}$, Donghui $\mathrm{Li}^{1}$, Karen Stegman ${ }^{1}$, Sabrina Ophir ${ }^{2}, \mathrm{Xia} \mathrm{Cao}^{1}$, Kristina

3 Atanasoff ${ }^{2,6}$, Reyna Lim ${ }^{1}$, Shreyas Kowdle ${ }^{2}$, Juan Manuel Carreño ${ }^{2}$, Laura Rivero-Nava ${ }^{1}$, Ariel Raskin ${ }^{2}$,

4 Elena Moreno ${ }^{2}$, Sachi Johnson ${ }^{1}$, Raveen Rathnasinghe ${ }^{2,6}$, Chin I Pai ${ }^{1}$, Thomas Kehrer ${ }^{2,6}$, Elizabeth Paz

5 Cabral $^{1}$, Sonia Jangra ${ }^{2}$, Laura Healy ${ }^{1}$, Gagandeep Singh ${ }^{2}$, Prajakta Warang ${ }^{2}$, Viviana Simon ${ }^{2,5,7,10}$, Mia

6 Emilia Sordillo ${ }^{10}$, Harm van Bakel ${ }^{11}$, Yonghong Liu' ${ }^{2}$, Weina Sun ${ }^{2}$, Lisa Kerwin ${ }^{1}$, Peter Palese ${ }^{2,7}$, John

7 Teijaro ${ }^{4}$, Michael Schotsaert ${ }^{2,5}$, Florian Krammer ${ }^{2,8}$, Damien Bresson ${ }^{1}$, Adolfo García-Sastre ${ }^{2,5,7,8,9}$, Yanwen

$8 \mathrm{Fu}^{1}$, Benhur Lee ${ }^{2}$, Colin Powers ${ }^{1}$, Thomas Moran ${ }^{2,3}$, Henry Ji ${ }^{1 \#}$, Domenico Tortorella ${ }^{2,3}$, Robert Allen ${ }^{1}$

\section{Affiliations:}

${ }^{1}$ Sorrento Therapeutics, Inc., San Diego, CA 92121

112 Department of Microbiology,

12 Icahn School of Medicine, Mount Sinai, New York, NY

${ }^{3}$ Center for Therapeutic Antibody Development, Drug Discovery Institute, Icahn School of Medicine, Mount Sinai, New York, NY

${ }^{4}$ Department of Immunology and Microbial Science, The Scripps Research Institute, La Jolla, CA 92037

${ }^{5}$ Global Health and Emerging Pathogens Institute, Icahn School of Medicine, Mount Sinai, New York, NY

${ }^{6}$ Graduate School of Biomedical Sciences, Icahn School of Medicine at Mount Sinai, New York, NY

${ }^{7}$ Department of Medicine, Division of Infectious Diseases, Icahn School of Medicine at Mount Sinai, New York, NY

${ }^{8}$ Department of Pathology, Molecular and Cell-Based Medicine, Icahn School of Medicine at Mount Sinai, New York, NY

${ }^{9}$ The Tisch Cancer Institute, Icahn School of Medicine, Mount Sinai, New York, NY

${ }^{10}$ Department of Pathology, Molecular and Cell based Medicine, Icahn School of Medicine, Mount Sinai, New York, NY 
bioRxiv preprint doi: https://doi.org/10.1101/2022.01.19.476998; this version posted January 20, 2022. The copyright holder for this preprint (which was not certified by peer review) is the author/funder. All rights reserved. No reuse allowed without permission. 
The continual emergence of SARS-CoV-2 variants of concern, in particular the newly emerged Omicron (B.1.1.529) variant, has rendered ineffective a number of previously EUA approved SARS-CoV-2 neutralizing antibody therapies. Furthermore, even those approved antibodies with neutralizing activity against Omicron are reportedly ineffective against the subset of Omicron variants that contain a R346K substitution, demonstrating the continued need for discovery and characterization of candidate therapeutic antibodies with the breadth and potency of neutralizing activity required to treat newly diagnosed COVID-19 linked to recently emerged variants of concern. Following a campaign of antibody discovery based on the vaccination of Harbour H2L2 mice with defined SARS-CoV-2 spike domains, we have characterized the activity of a large collection of Spike-binding antibodies and identified a lead neutralizing human IgG1 LALA antibody, STI-9167. STI-9167 has potent, broad-spectrum neutralizing activity against the current SARS-COV-2 variants of concern and retained activity against the Omicron and Omicron + R346K variants in both pseudotype and live virus neutralization assays. Furthermore, STI-9167 nAb administered intranasally or intravenously provided protection against weight loss and reduced virus lung titers to levels below the limit of quantitation in Omicron-infected K18-hACE2 transgenic mice. With this established activity profile, a cGMP cell line has been developed and used to produce cGMP drug product intended for use in human clinical trials. 


\section{INTRODUCTION}

60

61

62

63

64

65

The severe acute respiratory disease syndrome coronavirus 2 (SARS-CoV-2) pandemic has continued to significantly impact the health and lives of people around the globe ${ }^{1}$. To date, public health agencies have sought to combat infections leading to COVID-19 by relying on quarantine, social distancing, vaccination, and antiviral countermeasure strategies ${ }^{2,3}$. Despite these efforts, the continued spread of SARS-CoV-2 has led to the emergence of several variants of concern (VOCs) that have risen in prevalence worldwide ${ }^{2-7}$.

Each VOC encodes multiple changes in the amino acid sequence of the SARS-CoV-2 spike that can impact the neutralizing properties of manufactured SARS-CoV-2 neutralizing antibodies (nAbs) as well as nAbs elicited following vaccination or during the course of natural infection. Specifically, the Omicron VOC (B.1.1.529) live virus, when profiled in vitro using Vero cells expressing human ACE2 and human TMPRSS2 for susceptibility to nAbs currently authorized or approved for clinical use (AFCU nAbs), has been shown to be resistant to the neutralizing activities of REGN10987 (imdevimab), REGN10933 (casirivimab), LY-CoV555 (bamlanivimab), LYCoV016 (etesevimab), and CT-P59 (regdanvimab), at nAb concentrations $\leq 10 \mu \mathrm{g} / \mathrm{mL}\left(\mathrm{IC}_{50}\right)$, and remained susceptible to nAbs COV2-2130 (cilgavimab) and COV2-2196 (tixagevimab) tested as single $\mathrm{nAb}$ therapies or in combination ( $\left(\mathrm{C}_{50} \text { of } 43,126 \text {, and } 181 \mathrm{ng} / \mathrm{mL} \text {, respectively }\right)^{8-13}$. In live virus neutralization assays utilizing Vero cells overexpressing human TMPRSS2, S309 (sotrovimab) registered an $\mathrm{IC}_{50}$ of $373 \mathrm{ng} / \mathrm{mL}$, consistent with previously published activity in Omicron pseudovirus assays for this antibody. 
A subset (approximately $23 \%$ of Omicron sequences in GISAID recorded on outbreak.info) of

Omicron viruses encode an additional mutation in the SARS-CoV-2 spike at position R346K in the receptor binding domain (RBD) of the protein ${ }^{14-16}$. The R346K mutation was previously identified among the defining mutations of the SARS-CoV-2 Mu VOC ${ }^{7}$. Using Omicron + R346K pseudoviruses, neutralization potency was reported as substantially reduced for all tested AFCU nAbs, including COV2-2130, COV2-2196, and S309 5,17-20. Current antibodies in development,

85 including bebtelovimab and BRII-198 (romlusevimab), maintain activity in Omicron pseudotype neutralization assays ${ }^{12,21}$. BRII-198 displays substantially reduced neutralizing activity in assays using Omicron + R346K pseudoviruses while testing of bebtelovimab against the Omicron + R346K variant has not yet been reported ${ }^{12,21}$. As such, there is a continued need for discovery and development of nAbs that can provide potent immune protection against COVID-19 caused by pandemic VOCs presently infecting the global population.

In the early COVID-19 disease setting, intravenous (IV) administration of nAbs is an effective means of lessening progression and overall severity of disease ${ }^{18,22}$. As COVID-19 is a predominantly respiratory disease, exploration of alternative modes of antibody administration including intranasal (IN) delivery may provide an expedient means of delivering antibodies and increasing the respiratory tract bioavailability of anti-COVID-19 nAbs as well as augmenting the developing host-directed immune response to prevent exacerbation of clinical symptoms and hospitalization ${ }^{23-25}$. activity of STI-9167 against live viruses and pseudotype viruses representing the current catalog of SARS-CoV-2 variants, including the Omicron and Omicron +R346K variant. Additionally, we 
101 describe the protective effects of STI-9167 administered IV or IN in the K18ACE2 transgenic

102 mouse model of COVID-19 disease following challenge with either the WA-1 strain, Delta, or

103 Omicron VOC.

104 RESULTS

105 Generation of human anti-SARS-CoV-2 spike antibodies

106 To generate a panel of neutralizing human monoclonal antibodies against SARS-CoV-2, Harbour

$107 H 2 L 2^{\circledR}$ mice were immunized and boosted with a receptor binding domain (RBD) fusion protein

108 based on the original spike glycoprotein sequence from the Wuhan seafood market pneumonia

109 virus isolate (GenBank Accession\# MN908947) which was fused to a mouse Fc domain (Figure

110 1A). The sera from immunized mice were assessed for binding to 293ExpiF cells transfected

111 with SARS-CoV-2 spike cDNA (original Wuhan strain) using high-throughput flow cytometry

112 (Figure 1B). We observed that the serum from Mouse 1, 3, and 4 demonstrated a concentration

113 dependent and specific binding to 293ExpiF cells expressing SARS-CoV-2 spike. Given that

114 Mouse 3 and Mouse 4 had the highest titer humoral response against SARS-CoV-2 spike, the

115 spleens from these animals were used to generate hybridoma clones ${ }^{26}$. The hybridoma clones

116 (1,824 clones from Mouse 3 and 1,440 clones from Mouse 4) were screened for binding to

117 293ExpiF cells expressing SARS-CoV-2 spike by flow cytometry and RBD-spike (Wuhan) (Figure

118 1). A representative heat map for Mouse 4 fusion was generated to summarize the mean

119 fluorescence intensity (MFI) for each hybridoma clone (Figure 1C). In parallel, hybridoma clones

120 were subjected to an RBD ELISA validating the clones that bound to SARS-CoV-2 spike. We

121 identified 188 clones with a >5-fold MFI over untransfected cells and classified these as 
candidate SARS-CoV-2 binding antibodies. The supernatants from these clones were then evaluated in a high-throughput neutralization assay using the replication competent VsV reporter virus that utilizes SARS-CoV-2 spike (VsVCoV2-spike) as its envelope protein and expressing GFP as readout for infection ${ }^{27}$ (Figure 1C, Secondary Screening). Briefly, VsVCoV2-spike was preincubated with hybridoma supernatant (1:20) followed by infection of HEK-293 cells expressing TMPRSS2 for $24 \mathrm{hrs}$ and analyzed for GFP positive cells using flow cytometry. The \% infection was determined by assigning $100 \%$ infection with VsV CoV2-spike pre-incubated with hybridoma media alone. Thirty-eight clones that decreased infection $>50 \%$ were selected for further evaluation for neutralization by determining the $\mathrm{IC}_{50}$ values. For the hybridomas from the Mouse 3 fusion, 340 clones were found to bind to SARS-CoV-2 spike and 90 clones were found to have neutralization activity against VsVCoV2-spike. The selected neutralizing clones with $\mathrm{IC}_{50}$ values $<125 \mathrm{pM}$ were then examined for IgG isotypes and expanded for further analysis. Sequencing of the heavy chain from each hybridoma clone revealed diverse CDR3 lengths ranging from 10-20 aa in length (Figure 1D). Clones that were identical copies of each other were consolidated to a single candidate.

To identify the most effective human anti-SARS-CoV-2 spike neutralizing antibodies, we performed a VsV CoV2-spike neutralization assay (Figure 1D). Briefly, VsV CoV2-spike preincubated with increasing concentrations of antibody $(0-5 \mathrm{mg} / \mathrm{mL})$ was added to TMPRSS2-expressing HEK-293 cells and analyzed for GFP positive cells using flow cytometry.

VsV CoV2-spike preincubated with a control antibody was used as $100 \%$ infection. The various unique antibodies have a range of $\mathrm{IC}_{50}$ values from 5-1,000pM across the different antibody families. Collectively, we have identified a panel of unique human antibodies that bind to SARS- 
144 CoV-2 and effectively neutralize a reporter virus that utilizes the SARS-CoV-2 spike for entry into 145 human cells.

146 Candidate nAbs sequences were formatted as full-length human IgG1 antibodies and expressed

147 in Chinese hamster ovary $(\mathrm{CHO})$ cells for further characterization in vitro. It has been shown in

148 the context of multiple virus infections that virus-specific antibodies can lead to exacerbation of

149 disease symptoms through a process termed antibody dependent enhancement (ADE) ${ }^{28,29}$. To

150 reduce the risk of ADE resulting from administration of our lead candidate STI-9167, the IgG1 Fc

151 regions were modified by introducing specific amino acid substitutions (L234A, L235A [LALA])

15230,31 . The LALA Fc modification reduces binding affinity to the Fcy receptors while providing a

153 similar blockade to interactions between SARS-COV-2 and the angiotensin-converting enzyme 2

154 (ACE2) receptor expressed on susceptible cells in the lung and other organs ${ }^{32-34}$.

155 To determine the effects of variant specific spike S1 domain mutations within and outside the

156 RBD region of S1 on antibody binding, the affinity of STI-9167 and EUA-approved SARS-CoV-2

157 nAbs sotrovimab, cilgavimab, and tixagevimab were determined for monomeric WA-1 spike S1

158 subunit binding as well as VOC-derived S1 domains using surface plasmon resonance (SPR). Of

159 note, the $\mathrm{k}_{\mathrm{D}}$ of STI-9167 was measured as $6.20 \mathrm{nM}$ for the WA-1 isolate, $4.45 \mathrm{nM}$ for the Delta

160 variant, and $22.6 \mathrm{nM}$ for the Omicron variant. Binding kinetics for the Omicron variant were

161 compared between STI-9167, sotrovimab, cilgavimab, and tixagevimab. (Figure 2A, Table 1A

162 and Supplemental Figure 4). STI-9167 and cilgavimab had a similar association rate and

163 sotrovimab was approximately 5 -fold slower. The dissociation rate was slowest with sotrovimab 
approximately 2 -fold slower rate than cilgavimab. Tixagevimab binding to Omicron S1 domain monomer was insufficient to allow for quantitation.

In an effort to assess nAb binding to spike proteins in a native conformation, STI-9167 was tested for the binding of full-length spike protein expressed on the surface of transfected HEK293 cells. Cell-based binding studies demonstrated STI-9167 binds with similar efficiency to surface-expressed spike from the WA- 1 isolate $\left(\mathrm{EC}_{50}=0.025 \mu \mathrm{g} / \mathrm{mL}\right)$, Delta variant $\left(\mathrm{EC}_{50}=0.011\right.$ $\mu \mathrm{g} / \mathrm{mL})$, and the Omicron variant $\left(\mathrm{EC}_{50}=0.024 \mu \mathrm{g} / \mathrm{mL}\right)$, as well as the greater catalog of $\mathrm{VOC}$ spike protein (Figure 2B and Supplemental Figure 1). In general, the rank-order of binding efficiencies to surface-expressed spike for those nAbs considered in the SPR studies followed the same pattern as that determined for spike monomer binding, with the greatest concordance in binding efficiency seen between STI-9167 and cilgavimab (Table 1B and

Supplemental Figure 5). Of note, half-maximal binding of STI-9167 to the Omicron + R346K spike $\left(E_{50}=0.023 \mu \mathrm{g} / \mathrm{mL}\right.$ ) was equivalent to that measured for Omicron, suggesting that the epitope recognized by STI-9167 is preserved in the context of Omicron + R346K as compared to epitopes engaged by cilgavimab, which displayed reductions in Omicron + R346K spike binding of over 60 -fold as compared to $\mathrm{EC}_{50}$ values in assays targeting Omicron spike. Based on the spike S1 and full-length spike protein binding data, STI-9167 was further profiled to determine the potency of virus neutralization and the breadth of neutralizing protection this antibody provided against SARS-CoV-2 variants of concern in vitro.

Virus pseudotypes were used to determine the neutralization potency (IC $\left.C_{50}\right)$ of STI-9167 against an index virus generated with a spike protein that carries a single D614G (VSV D614G-spike) mutation as compared to the WA-1 spike protein ${ }^{35}$. To approximate conditions found in the 
setting of human SARS-CoV-2 infection, pseudovirus assays were carried out using HEK293 cells pseudotypes as well as for the VSV ${ }^{\text {D614G-spike }}$ pseudotype are detailed in Table 1C.

The potency of STI-9167, cilgavimab, and tixagevimab was further characterized in live virus neutralization assays utilizing Vero cells . Neutralizing activity was determined following infection with WA-1 strain or Omicron variant and compared to EUA approved antibodies

(Table 1D and Supplemental Figure 3). In keeping with the results from pseudovirus assays, we with an $\mathrm{IC}_{50}$ of $54.29 \mathrm{ng} / \mathrm{ml}$ against live Omicron variant virus (Figure 2D). Neutralization 
Bioavailability

209

210

211

212

213

214

215

216

217

218

219

220

221

222

223

224

225

226

227

228

229

The biodistribution of STI-9167 was evaluated following delivery by either the intravenous or intranasal route. These studies illustrate the potential effects of delivery route on the timing of antibody exposure in the lung tissue and sera of treated mice. Following IV treatment at a dose level of $0.5 \mathrm{mg} / \mathrm{kg}$, STI-9167 was detected in the serum, spleen, lungs, small intestine, and large intestine of most animals. Detected levels in the serum following IV dosing at the $0.5 \mathrm{mg} / \mathrm{kg}$ dose averaged $6.2 \mu \mathrm{g} / \mathrm{mL}$, while STI-9167 was undetected in lung lavage material at each of the IV doses tested, (Figure 3A, upper left panel). Upon processing of lung tissue, antibody was detected at a mean concentration of $0.4 \mathrm{ng} / \mathrm{mg}$ of tissue in the $0.5 \mathrm{mg} / \mathrm{kg}$ IV dose group. Lower IV doses of STI-9167 did not lead to a statistically significant difference in antibody detected in lung tissue as compared to untreated animals. Antibody levels in the spleen reached an average concentration of $0.2 \mathrm{ng} / \mathrm{mg}$ of tissue within 24 hours of IV dosing at $0.5 \mathrm{mg} / \mathrm{kg}$. Similarly, antibody was detectable in a majority of both the small and large intestines only at the highest dose level, with average concentrations of 0.14 and $0.07 \mathrm{ng} / \mathrm{mg}$ of tissue, respectively (Figure 3A, upper right panel).

Following intranasal (IN) administration of STI-9167, the concentration of antibody in the serum at 24 hours reached an average value of $0.054 \mu \mathrm{g} / \mathrm{mL}$ in the $0.5 \mathrm{mg} / \mathrm{kg}$ dose group. As compared to IV treated animals at the $0.5 \mathrm{mg} / \mathrm{kg}$ dose, STI-9167 administered IN resulted in a 114-fold lower concentration of antibody in serum at the 24-hour timepoint. In contrast to the observed reductions in IN serum nAb levels vs. those following IV nAb administration, STI-9167 concentrations in lung lavage samples following IN dosing reached average concentrations of $0.18 \mu \mathrm{g} / \mathrm{mL}$ in the $0.5 \mathrm{mg} / \mathrm{kg}$ group, a 9-fold increase over lung lavage $\mathrm{nAb}$ levels observed 
230 following IV delivery of the $0.5 \mathrm{mg} / \mathrm{kg}$ dose, confirming that lung lavage materials can more

231 efficiently collect drugs delivered through the airway than those delivered IV. In lung tissue

232 samples 24 hours following the $0.5 \mathrm{mg} / \mathrm{kg}$ IN dose, STI-9167 was detected at an average

233 concentration of $0.173 \mathrm{ng} / \mathrm{mg}$ of tissue, similar to those levels recorded in IV-treated animals at

234 the same dose level. STI-9167 levels in spleen, small and large intestine at all IN dose levels

235 tested did not rise to concentrations above background (Figure 3B).

236 Overall, IN delivery of STI-9167 led to lower serum concentrations, increased lung lavage

237 concentrations, and similar tissue concentrations in the spleen, lungs, small intestine, and large

238 intestine when compared to IV delivery. This suggests that IN administration serves to increase

239 the amount of antibody in the pulmonary lavage material, potentially allowing for more

240 efficient neutralization of respiratory virus particles present in the extravascular spaces along

241 the respiratory tract during the initial stages of infection.

\section{$242 \quad$ Pharmacokinetics}

243 To characterize STI-9167 pharmacokinetic parameters following intranasal dosing, antibody

244 levels in CD-1 mouse lung tissue lysates and serum were quantified at designated timepoints

245 spanning a total of $336 \mathrm{~h}$ using a human antibody detection ELISA assay. In this assay the

246 background concentration was on average $16.8 \mathrm{ng} / \mathrm{mL}$ based on measurements obtained using

247 pre-dose samples. Following IN administration of STI-9167, the antibody concentration was

248 quantifiable for most of the animals at the $336 \mathrm{~h}$ timepoint in both the lungs and serum (Figure

$2493 \mathrm{C})$. The $C_{\max }$ value of STI-9167 in the lungs was measured at 1.5 hours ( $\left.T_{\max }\right)$ post-

250 administration at a value of $43 \mu \mathrm{g} / \mathrm{mL}$. In the lungs following IN administration, STI-9167 
251 exhibited an apparent terminal half-life $\left(T_{1 / 2}\right)$ of $26.6 \mathrm{~h}$. Kinetics of STI-9167 exposure in the

252 lungs following IN administration contrasted with the slower rate of antibody accumulation in

253 the serum of treated mice (Figure 3C). Antibody was first detected in the serum at 1.5 hours

254 post-administration and the $C_{\max }$ of $456 \mathrm{ng} / \mathrm{mL}$ was reached at the $168 \mathrm{~h}$ timepoint $\left(T_{\max }\right.$ ),

255 although consideration of the standard deviations in values measured among animals on or

256 between the $72 \mathrm{~h}$ and $168 \mathrm{~h}$ timepoints suggests that the $T_{\max }$ may have occurred as early as 72

257 hours post-administration. Antibody levels remained relatively constant in serum over the

258 period spanning $24-336 \mathrm{~h}$, which is in keeping with the calculated STI-9167 serum half-life

259 observed following IV STI-9167 administration in CD-1 mice (data not shown). The total

260 systemic antibody exposure ( $\left.A \cup C_{\text {last }}\right)$ was greater than 5-fold higher in the lungs than in the

261 serum of IN-treated mice ( $A \cup C_{\text {last }}$ were 594,705 and 105,149 $\mathrm{h} * \mathrm{ng} / \mathrm{mL}$ respectively).

262 Treatment using IV or IN administered STI-9167 in the K18-hACE2 transgenic mouse model of

263 COVID-19

264 SARS-CoV-2 pathogenesis in the K18-ACE2 transgenic model of COVID-19 respiratory disease

265 provides a tractable means of assessing nAb activity in a preclinical model of respiratory disease

$263^{36-38}$. The clinical signs and histological markers of pathogenesis in this model include weight

267 loss over the first four to five days post-infection and the presence of microscopic lesions in the

268 infected lungs ${ }^{37-40}$. Peak virus lung titers are typically detected by day 5 post-infection, but the

269 timing and peak amplitude of replication in the lungs can vary depending on the specific VOC

270 used to challenge the mice ${ }^{39}$. The breadth of protection provided by STI-9167 was established

271 by treating mice following virus challenge with $1 \times 10^{5}$ half-maximal tissue culture infectious

272 dose $\left(\mathrm{TCID}_{50}\right)$ of the WA-1 strain, the Delta variant, or the Omicron variant. Animals treated 
273 with isotype control antibody lost weight in each experiment, with the average percentage

274 weight reduced to $91.3 \%$ with WA-1 strain, $89 \%$ with Delta variant, and $94.7 \%$ with Omicron

275 variant (Figure 4B, 4E) as compared to average Day 0 weights in each group. To determine the

276 effects of the route of administration on the degree of protection conferred by treatment with

277 STI-9167 at doses ranging from 5 to $20 \mathrm{mg} / \mathrm{kg}$, antibody was administered by either intravenous

278 injection (Figure 4A) or intranasal instillation (Figure 4D). At a dose level of $5 \mathrm{mg} / \mathrm{kg}$,

279 administration of STI-9167 to K18-hACE2 mice by either the IV or IN route provided protection

280 against weight loss caused by WA-1 strain, Delta variant, and Omicron variant (Figure 4B, 4E).

281 Virus replication in the lungs, quantified on day 4 post-infection, was approximately $2.5 \times 10^{6}$,

$2822.1 \times 10^{4}$, and $4.5 \times 10^{2} \mathrm{TCID}_{50} / \mathrm{g}$ on average in isotype control-treated mice infected with WA-1,

283 Delta variant, or Omicron variant, respectively. Following infection by each of the SARS-CoV-2

284 challenge viruses, lung virus titers in mice treated with STI-9167 were reduced to levels below

285 the limit of quantification, independent of the nAb dosing route or the nAb dose level (Figure

286 4C, 4F).

287 DISCUSSION

288 Use of antibody discovery platforms that do not require material derived from infected

289 individuals, such as the vaccination strategy employed here or the screening of established

290 antibody libraries, can provide a preemptive means of addressing the challenges presented by

291 pandemic threat pathogens ${ }^{14,41}$. The production of human antibodies in transgenic animals has

292 several advantages including in vivo affinity maturation, increased diversity, and clonal selection

293 for antibody optimization ${ }^{42}$. Thus, the generation of antibodies to specific protein domains

294 allows for the development of highly reactive and effective antibody therapeutics. 
295 The pool of antibodies we identified following vaccination of mice with an SARS-CoV-2 RBD

296 protein based on the Wuhan spike protein sequence includes a candidate with potent

297 neutralizing activity against many SARS-CoV-2 variants of concern that have emerged in the

298 past two years of the pandemic. Our antibody binding studies and virus neutralization assays

299 have provided clear evidence of the broad and potent neutralizing activity of STI-9167 toward

300 those VOCs identified in the early period of the pandemic as well as those VOCs currently

301 impacting public health, including Delta and Omicron. Following demonstration of neutralizing

302 activity against the parental Omicron variant, we have extended our activity profiling studies to

303 include assessment of the Omicron + R346K subvariant, a virus that is currently represented in a

304 reported $23 \%$ of sequences submitted to GISAID ${ }^{14-16}$. The frequency of Omicron virus

305 sequences containing the R346K substitution has risen steadily since the first reports in

306 November of 2021 describing detection of the Omicron variant ${ }^{43-45}$. Using virus pseudotypes,

307 we have demonstrated durable STI-9167 activity against the Omicron +R346K subvariant. In

308 addition, we described neutralizing activity against the Mu variant, which also encodes the

309 R346K spike substitution ${ }^{7}$. The Omicron and Mu variants constitute divergent variants of SARS-

310 CoV-2, and it appears that the R346K substitution is not sufficient in either of these contexts to

311 provide a means of resistance to the neutralizing effects of STI-9167.

312 Our studies of nAb STI-2020 previously demonstrated the protective efficacy of IN-administered 313 antibodies in the context of SARS-CoV-2 preclinical models of pathogenesis ${ }^{46}$. Previous work in

314 preclinical models of respiratory virus pathogenesis support the use of IN-administered IgG and

$315 \operatorname{IgA}$ mAbs in prophylactic and therapeutic dosing regimens ${ }^{24,47-50}$. In the current report, we

316 described the protective effects of STI-9167 delivered by either the IN or the IV route to animals 
317 infected with WA-1 strain, Delta, or Omicron variants. As evidenced in recently reported

318 preclinical studies of Omicron pathogenesis as well as our experiments, the severity of clinical

319 signs and the amount of virus replication in the lungs following Omicron infection was reduced

320 in comparison to that following infection with the WA-1 strain or the Delta variant ${ }^{51-53 .}$

321 Independent of the challenge virus used, at a dose level of $5 \mathrm{mg} / \mathrm{kg}$, IN treatment with STI-9167

322 in K18 ACE2 transgenic mice 12 hours following infection provided protection against the

323 weight loss observed in control animals and also reduced virus lung titers to below the level of

324 quantitation. Phase 1 clinical studies with STI-2099 (plutavimab) have demonstrated the safety

325 of nAb delivered as formulated liquid drops to the upper airways. A Phase 2 study has

326 completed enrollment in the US, and additional Phase 2 studies are ongoing in Mexico and the

327 United Kingdom. Based on the favorable in vivo potency and physicochemical profile of STI-

3289167, cGMP drug product has been prepared in preparation for similar anticipated clinical

329 studies of STI-9167 administered IV or as intranasal drops (STI-9199).

MATERIALS AND METHODS

$331 \quad$ Immunizations and Hybridoma Generation

332 To generate human antibodies, Harbour $H 2 L 2^{\circledR}$ human antibody transgenic mice (Harbour

333 BioMed, Cambridge, MA) were utilized under a collaboration between the Icahn School of

334 Medicine at Mount Sinai and Harbour BioMed. The H2L2 transgenic mouse is a chimeric

335 transgenic mouse containing the human variable gene segment loci of the heavy and kappa

336 antibody chains along with the rat heavy and kappa constant gene segment loci, producing a

337 mouse with normal B cell homeostasis and effector functions, while also producing antibodies 
338 that represent the typical diversity observed in human antibody immunity ${ }^{54}$. Immunizations were done on eight- to twelve-week-old H2L2 mice interperitoneally with 50-100 $\mu \mathrm{g}$ of a

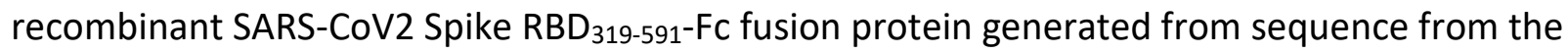
original Wuhan seafood market pneumonia virus isolate (GenBank Accession\# MN908947) and

342 cloned in-frame into pcDNA vectors containing human IgG1 and mouse IgG2a Fc tags (GenScript

343 USA Inc., Piscataway, NJ). Each mouse received a prime followed by 2 boosts, and blood was

344 collected from the submandibular vein two weeks after each boost to monitor titer of sera

345 antibodies. Following sera binding and neutralization analysis, two mice were selected for

346 hybridoma fusion and received two final boosts consisting of 50-100 $\mu \mathrm{g}$ of the RBD protein at -

3475 and -2 days before being euthanized by IACUC approved methods with spleens harvested and

348 a final bleeding collected for sera analysis ("fusion sera"). The spleens were processed to single

349 cell suspension and hybridomas were generated using the standard protocol. Briefly, individual

350 B cell clones were grown on soft agar and selected for screening using a robotic ClonaCell Easy

351 Pick instrument (Hamilton/Stem Cell Technology). Individual clones were expanded, and the

352 supernatants were used to screen for binding, neutralization and ACE2 competition assays. All

353 animal studies were approved by the Icahn School of Medicine Institutional Animal Care and

354 Use Committee (IACUC).

355 Hybridoma Screening: Expi293F cells were transiently transfected to express SARS-CoV-2 spike

356 (Wuhan) using Lipofectamine 3000 (L3000001, Thermo Fisher) and then incubated with

357 supernatant from the hybridoma cell lines from each fusion. Binding was detected using an

358 anti-rat IgG-APC detection antibody and samples were run on a high-throughput flow

359 cytometer (Intellicyte High Throughput Flow Cytometer [Intellicyte Corp., Albuquerque, NM]. 
360 Samples were compared to controls of fusion sera, unimmunized "normal" mouse sera, and an

361 in-house generated anti-SARS1\&2 Spike mouse monoclonal 2B3E5 at $1 \mu \mathrm{g} / \mathrm{mL}$. Cells with a high

362 mean fluorescence intensity (MFI) were identified using FlowJo software (Tree Star, Inc.) and

363 graphed using GraphPad Prism to create a heat map based on mean fluorescence intensity.

364 ELISA: Immulon 4 HBX high binding clear flat bottom 96 well plates (ThermoFisher) were coated

365 with SARS-CoV2 Spike $\mathrm{RBD}_{319-591}-\mathrm{Fc}$ fusion at $5 \mu \mathrm{g} / \mathrm{ml}$ in $1 x P B S$ overnight at $4{ }^{\circ} \mathrm{C}$ followed by

366 washing. Washing with 1XPBS was done between each step in triplicate using a Biotek ELX405

367 MultiPlate Washer (Biotek, Winooski, VT). Plates were blocked for two hours in blocking

368 solution (1XPBS, $0.5 \%$ BSA). Supernatants from the hybridomas were then added and allowed

369 to incubate for one hour at room temperature followed by the addition of goat anti-rat IgG

370 (heavy chain specific)-HRP (Jackson ImmunoResearch) at a 1:5,000 dilution in blocking solution

371 for one hour. ABTS substrate solution (ThermoFisher) was added and allowed to incubate for 5-

37210 minutes at room temperature, protected from light. Absorbance at 450nm was measured

373 using a Biotek Synergy HT Microplate Reader. Fusion sera, normal mouse sera, and 2B3E5 mAb

$374 \quad(0.5-1 \mu \mathrm{g} / \mathrm{ml})$ were used as controls.

375 Neutralization: Prior to neutralization, hybridoma supernatants grown in SFM (sera free

376 hybridoma media) (Invitrogen) were quantitated using an Octet Red96 by diluting supernatants

$377 \quad 1: 5$ and $1: 10$ in sera free media and measured for binding against the Anti-Murine IgG

378 Quantitation (AMQ) Biosensors (with cross reactivity to rat lgG Fc) on an Octet Red 96 BLI

379 Instrument (SartoriusAG, Goettingen, Germany). Results were compared to in-lab derived

380 purified rat IgG standards diluted in SFM in the range of 0.5-50 $\mu \mathrm{g} / \mathrm{ml}$. For neutralization, VsV- 
SARS-spike GFP-expressing reporter virus was pre-incubated with mouse sera $(1: 100-1,200)$, expressing human ACE2 and Transmembrane Serine Protease-2 overnight at $37^{\circ} \mathrm{C}, 5 \% \mathrm{CO}_{2}{ }^{27}$.

The cells were resuspended in cold FACS buffer and analyzed by flow cytometry (Intellicyte based on MFI.

\section{Sequencing and Humanizing of Antibodies}

Sequence of the human variable heavy and kappa chains were obtained by using SMARTer $5^{\prime}$

RACE technology (Takara Bio USA) adapted for antibodies to amplify the variable genes from

incubation with the SMARTer II A Oligonucleotide and SMARTscribe reverse transcriptase. 
and DNA was synthesized and cloned in-framed into pcDNA-based vectors containing a human IgG1 constant region and a human kappa light chain constant region (GenScript USA Inc.,

Piscataway, NJ).

Synthesis of Comparison Antibodies

Antibody expression vector construction and antibody transient expression and purification were done following standard protocols. Briefly, heavy chain and light chain variable domain genes were designed by coding the amino acid sequences of an antibody using codon table of Cricetulus griseus for $\mathrm{CHO}$ (Chinese hamster ovarian) cells as expression host. The heavy chain and light chain variable domain gene fragments with flanking sequences for infusion cloning were synthesized by IDT (Integrated DNA technologies, San Diego), and cloned into a mammalian expression vector with built-in IgG1 constant domain and/or light chain constant

414 domain sequences. The expression vectors were confirmed by DNA sequencing. CHO-S cells in 415 exponential phase at a cell density of $10^{6}$ cells $/ \mathrm{ml}$ with viability of $\geq 93 \%$, were co-transfected 416 with both heavy and light chain expression plasmids of the target antibody. The transfection 417 complex was formed between DNA and PEI (polyethylenimine). Each antibody was harvested 418 by centrifuging the culture to pellet and remove the cells $10-14$ days after the transfection. The 419 supernatant was processed with a protein A column, and the Protein A bound antibody was 420 eluded with low pH glycine buffer. Purity of the antibodies were annualized by SDSPAGE to be 421 more than 95\%.

422 Reference for antibody sequences is as follows; Sotrovimab 
424 (https://www.genome.jp/entry/D11993) (https://www.genome.jp/entry/D11994)Antibody

425 characterization

426 Kinetic interactions between the antibodies and his-tagged antigen proteins were measured at

427 room temperature using Biacore T200 surface plasmon resonance (GE Healthcare). Anti-human

428 fragment crystallizable region (Fc region) antibody was immobilized on a CM5 sensor chip to

429 approximately 8,000 resonance units (RU) using standard N-hydroxysuccinimide/N-Ethyl-N'-(3-

430 dimethylaminopropyl) carbodiimide hydrochloride (NHS/EDC) coupling methodology. The

431 antibody $(1.5 \mu \mathrm{g} / \mathrm{mL})$ was captured for 60 seconds at a flow rate of $10 \mu \mathrm{L} /$ minute. The SARS-

432 CoV-2 Spike S1, SARS-CoV-2 (2019-nCoV) Spike S1- B.1.1.7 lineage mut (HV69-70 deletion, Y144

433 deletion, N501Y, A570D, D614G, P681H)-His and SARS-CoV-2 (2019-nCoV) Spike S1- B.1.351

434 lineage mut (K417N, E484K, N501Y, D614G)-His proteins were run at six different dilutions in a

435 running buffer of $0.01 \mathrm{M}$ HEPES pH 7.4, $0.15 \mathrm{M} \mathrm{NaCl}, 3 \mathrm{mM}$ EDTA, 0.05\% v/v Surfactant P20

436 (HBS-EP+). All measurements were conducted in HBS-EP+ buffer with a flow rate of 30

$437 \mu \mathrm{L} /$ minute. The affinity of antibody was analyzed with BIAcore T200 Evaluation software 3.1. A

$438 \quad$ 1:1 (Langmuir) binding model is used to fit the data.

$439 \quad$ Cell based Spike binding assay

440 Mammalian expression vectors were constructed by cloning of the synthesized gene fragments

441 encoding SARS-CoV-2 Spike variant proteins (see attached table indicating mutations

442 introduced into wild type [WA-1 strain] spike protein sequence). HEK293 cells were transfected

443 using FuGeneHD transfection reagent according to manufacturer's protocol (Promega, Cat \#

444 E2311). 48 hours post-transfection, cells were harvested using enzyme free cell dissociation 
445 buffer (ThermoFisher, Cat \#13151014.), washed once and resuspended in FACS buffer (DPBS +

$4462 \% \mathrm{FBS})$ at $2 \times 10^{6}$ cells/mL. For antibody binding to the cells expressing the Spike proteins, the

447 cells were dispensed into wells of a 96-well V bottom plate ( $40 \mu \mathrm{L}$ per well), and an equal

448 volume of $2 x$ final concentration of serially-diluted anti-S1 antibody solution was added. After

449 incubation on ice for 45 minutes, the cells were washed with 2 times of $150 \mu \mathrm{L}$ FACS buffer.

450 Detection of bound antibody was carried out by staining the cells with $50 \mu \mathrm{L}$ of 1:500 diluted

451 APC AffiniPure $\mathrm{F}\left(\mathrm{ab}^{\prime}\right)_{2}$ Fragment (Goat Anti-Human IgG $(\mathrm{H}+\mathrm{L})$. Jackson ImmunoResearch, Cat\#

452 109-136-4098) for 20 minutes on ice. The cells were washed once with $150 \mu L$ FACS buffer and

453 analyzed on IntelliCyt iQue ${ }^{\circledR}$ Screener (Sartorius) flow cytometry. Mean fluorescent intensity

454 values were obtained from the histograms. A sigmoidal four-parameter logistic equation was

455 used for fitting the MFI vs. mAb concentration data set to extract EC50 values (GraphPad Prism

$456 \quad$ 8.3.0 software).

\begin{tabular}{|l|l|}
\hline variant & Mutations (and mutations in RBD from 333 to 526) \\
\hline British B1.1.7 variant & D1118H \\
\hline South African B1.351 & L18F, D80A, D215G, deletion of 242-244, R246I, K417N, E484K, N501Y, D614G, \\
variant & A701V \\
\hline Brazilian and Japanese & L18F, T20N, P26S, D138Y, R190S, K417T, E484K, N501Y, H655Y, T1027I \\
P1 variant & \\
\hline
\end{tabular}




\begin{tabular}{|c|c|}
\hline New York variant & L5F, T95I, D253G, E484K, D614G, A701V \\
\hline $\begin{array}{l}\text { South California } \\
\text { variant }\end{array}$ & S13I, W152C, L452R \\
\hline Mexican variant & T478K, D614G \\
\hline $\begin{array}{l}\text { Indian variant, sub- } \\
\text { lineage B1.617.1 }\end{array}$ & T95I, G142D, E154K, L452R, E484Q, D614G, P681R, Q1071H, H1101D \\
\hline $\begin{array}{l}\text { Indian variant, sub- } \\
\text { lineage B1.617.2 }\end{array}$ & $\begin{array}{l}\text { T19R, T95I, G142D, deletion of 157-158, A222V, L452R, T478K, D614G, P681R, } \\
\text { D950N }\end{array}$ \\
\hline Omicron & $\begin{array}{l}\text { A67V, del69-70, T95I, G142D, del143-145, N211D, del212, G339D, S371L, S373P, } \\
\text { S375F, K417N, N440K, G446S, S477N, T478K, E484A, Q493R, G496S, Q498R, } \\
\text { N501Y, Y505H, T457K, D614G, H665Y, N679K, P681H, N764K, D796Y, N856K, } \\
\text { Q954H, N969K, L981F }\end{array}$ \\
\hline Omicron + R346K & \\
\hline
\end{tabular}

\section{Cells and Viruses}

460 Vero E6 cells were maintained in Dulbecco's modified Eagle's medium (DMEM, Corning, NY)

461 supplemented with $10 \%$ fetal bovine serum (FBS, Thermo Fisher Scientific, MA), 1\% penicillin-

462 streptomycin, and L-glutamine. The P3 stock of the SARS-CoV-2 USA/WA-1/2020, 202001, 
USA/CA-CDC5574/2020 and, MD-HP01542/2021 isolates were obtained from The World stocks were stored at $-80^{\circ} \mathrm{C}$ under BSL3 conditions.

BHK21 cells (ATCC \#CCL-10) were maintained in DMEM/F12 media (Thermo Fisher \#21041025)

468 supplemented with 10\% fetal bovine serum (Omega Scientific \#FB-02) and 5\% tryptose phosphate broth 469 (Thermo Fisher \#18050039). BHK21/WI-2 cells (Kerafast \#EH1011) were maintained in DMEM (Thermo

470 Fisher \#11965092) supplemented with 5\% fetal bovine serum. 293-ACE2 cells were maintained in DMEM

471 supplemented with 10\% fetal bovine serum and $200 \mu \mathrm{g} / \mathrm{mL}$ G418 (Invivogen \#ant-gn-2). HEK-Blue 293

472 hACE2-TMPRSS2 cells (Invivogen \#hkb-hace2tpsa) were maintained in DMEM supplemented with 10\%

473 fetal bovine serum, $0.5 \mu \mathrm{g} / \mathrm{mL}$ Puromycin (Invivogen \#ant-pr-1), $200 \mu \mathrm{g} / \mathrm{mL}$ Hygromycin-B (Invivogen

$474 \quad$ \#ant-hg-1), and $100 \mu \mathrm{g} / \mathrm{mL}$ Zeocin (Invivogen \#ant-zn-1).

475 SARS-COV-2 viruses were obtained from BEl resources (Washington strain NR-52281; Alpha

476 variant NR-54000; Beta Variant NR-54009; Gamma variant NR-54982; Delta variant NR- 55611

477 or NR-55672; Lambda variant NR- 55654: Omicron Variant NR-65461.) VeroE6 monolayers were

478 infected at an MOI of 0.01 in $5 \mathrm{~mL}$ virus infection media (DMEM + 2\% FCS +1X Pen/Strep).

479 Tissue culture flasks were incubated at $36^{\circ} \mathrm{C}$ and slowly shaken every 15 minutes for a 90-

480 minute period. Cell growth media $(35 \mathrm{~mL})$ was added to each flask and infected cultures were

481 incubated at $36^{\circ} \mathrm{C} / 5 \% \mathrm{CO} 2$ for 48 hours. Media was then harvested and clarified to remove

482 large cellular debris by room temperature centrifugation at 3,000 rpm. 
484 The day before infection, $2 \times 10^{4}$ Vero E6 cells were plated to 96 -well plates and incubated at 37

$485{ }^{\circ} \mathrm{C}, 5 \% \mathrm{CO}_{2}$. Monoclonal antibodies were 2 -fold serially diluted in infection media (DMEM+2\%

486 FBS). Sixty microliters of diluted samples were incubated with $200 \mu \mathrm{L}$ of $50 \%$ tissue culture

487 infective doses $\left(\mathrm{TCID}_{50}\right)$ of SARS-CoV-2 in $60 \mu \mathrm{L}$ for $1 \mathrm{~h}$ at $37^{\circ} \mathrm{C}$. One-hundred microliters of the

488 antibody/virus mixture were subsequently used to infect monolayers of Vero E6 cells grown on

48996 -well plates. Cells were fixed with $10 \%$ formalin and stained with $0.25 \%$ crystal violet to

490 visualize cytopathic effect (CPE). The neutralizing concentrations of monoclonal antibodies

491 were determined by complete prevention of CPE.

492

Plasmids

All SARS-CoV-2 Spike constructs for pseudotype generation were expressed from plasmid

pCDNA3.1 (ThermoFisher \#V79020). Codon optimized SARS-CoV-2 Wuhan Spike carrying the

D614G amino acid change (Sino Biological \#VG40589-UT(D614G)) was modified to remove the

last 21 amino acids at the C-terminus (Spike $\Delta 21)$ and was used as the parental clone. Amino

497 acid changes for each variant are as follows. Alpha: $\Delta 69-70, \Delta 144, N 501 Y, A 570 D, D 614 G$,

498 P681H, T716I, S982A, and D1118H. Beta: D80A, D215G, $4242-244$, K417N, E484K, N501Y,

499 D614G, and A701V. Epsilon: S13I, W152C, L452R, and D614G. Kappa: G142D, E154K, L452R,

500 E484Q, D614G, P681R, Q1071H, and H1101D. Delta: T19R, G142D, L156-157, R158G, L452R,

501 T478K, D614G, P681R, and D950N. Delta Plus: T19R, G142D, $1156-157$, R158G, K417N, L452R,

502 T478K, D614G, P681R, and D950N. Gamma: L18F, T20N, P26S, D138Y, R190S, K417T, E484K,

503 N501Y, D614G, H655Y, and T1027I. Zeta: E484Q, F565L, D614G, and V1176F. Lambda: G75V,

504 T76I, R246N, $\Delta 247-253$, L452Q, F490S, D614G, and T859N. B.1.1.318: T95I, $\Delta$ Y144, E484K,

505 D614G, P681H, and D796H. Mu: T95I, Y144T, Y145S, ins146N, R346K, E484K, N501Y, D614G, 
P681H, and D950N. Omicron: A67V, del69-70, T95I, G142D, del143-145, N211D, del212, G339D,

S371L, S373P, S375F, K417N, N440K, G446S, S477N, T478K, E484A, Q493R, G496S, Q498R,

N501Y, Y505H, T457K, D614G, H665Y, N679K, P681H, N764K, D796Y, N856K, Q954H, N969K,

509 L981F

VSV-Spike pseudotype generation

511 To generate each Spike pseudotyped VSV, 1.2E6 BHK21 cells were nucleofected with $2 \mu \mathrm{g}$ of

512 Spike plasmid using an Amaxa Nucleofector II with cell line kit L (Lonza \#VCA-1005) and

513 program A-031. Cells were plated to one well of a 6-well dish and incubated overnight at 37

$514{ }^{\circ} \mathrm{C} / 5 \% \mathrm{CO}_{2}$. The next day, cells were transduced with G-Pseudotyped $\Delta \mathrm{G}$-luciferase (G* $\Delta \mathrm{G}$ -

515 luciferase) rVSV (Kerafast \#EH1025-PM) at MOI 4 for 1 hour at $37^{\circ} \mathrm{C} / 5 \% \mathrm{CO}_{2}$. Cells were rinsed

516 twice with DPBS (Corning \#21-031-CM), $2 \mathrm{~mL}$ of fresh media added, and incubated for 24-44

517 hours at $37^{\circ} \mathrm{C} / 5 \% \mathrm{CO}_{2}$. Supernatants were collected, spun at $300 \mathrm{~g}$ for 5 minutes at room

518 temperature, aliquoted and stored at $-80^{\circ} \mathrm{C}$. Pseudotypes were normalized for luciferase

519 expression by incubating with $1 \mu \mathrm{g} / \mathrm{mL}$ anti-VSV-G clone 8G5F11 (Millipore \#MABF2337) for 30

520 minutes at room temperature followed by transduction of $293-A C E 2$ cells. G* $\Delta G$-luciferase VSV

521 of known titer was used as the standard. Transduced cells were incubated for 24 hours, $40 \mu \mathrm{L}$ of

522 ONE-Glo reagent (Promega \#E6110) added and luminescence measured using a Tecan Spark

523 plate reader.

Pseudotype virus neutralization assays

525 HEK-Blue 293 hACE2-TMPRSS2 cells were plated to white-walled 96-well plates at 40K cells/well 526 and incubated at $37^{\circ} \mathrm{C} / 5 \% \mathrm{CO}_{2}$. The next day, pseudotyped VSV was incubated with anti-spike 
527 (concentration as indicated) and anti-VSV-G $(1 \mu \mathrm{g} / \mathrm{mL})$ antibodies for 30 minutes at room

528 temperature and added to the HEK-Blue 293 hACE2-TMPRSS2 cells in triplicate. Transduced

529 cells were incubated for 24 hours, $40 \mu \mathrm{L}$ of ONE-Glo reagent (Promega \#E6110) added and

530 luminescence measured using a Tecan Spark plate reader. The percent inhibition was calculated

531 using 1-([luminescence of antibody treated sample]/[average luminescence of untreated

532 samples]) $\times 100$. Absolute IC50 was calculated using non-linear regression with constraints of

533100 (top) and 0 (baseline) using GraphPad Prism software. The average of triplicate samples in

534 each of at least 3 independent experiments were included in the analyses. Negative value

535 slopes were assigned IC50 of $>10 \mu \mathrm{g} / \mathrm{mL}$. IC80 values were calculated using non-linear regression

536 with $\mathrm{F}=80$ and constraints of 100 (top) and 0 (bottom). For antibody comparison experiments, data for

537 Omicron and Omicron+R346K is an average of 2 independent experiments.

538 Affinity measurements

539 Kinetic interactions between the antibodies and his-tagged antigen proteins were measured at

540 room temperature using Biacore T200 surface plasmon resonance (GE Healthcare). Anti-human

541 fragment crystallizable region (Fc region) antibody was immobilized on a CM5 sensor chip to

542 approximately 8,000 resonance units (RU) using standard N-hydroxysuccinimide/N-Ethyl-N'-(3-

543 dimethylaminopropyl) carbodiimide hydrochloride (NHS/EDC) coupling methodology. The

544 antibody $(0.5-1 \mu \mathrm{g} / \mathrm{mL})$ was captured for 60 seconds at a flow rate of $10 \mu \mathrm{L} / \mathrm{minute}$. Each of the

545 following five variants of SARS-CoV-2 Spike S1 proteins were run at six different dilutions in a

546 running buffer of $0.01 \mathrm{M} \mathrm{HEPES} \mathrm{pH} \mathrm{7.4,} 0.15 \mathrm{M} \mathrm{NaCl}, 3 \mathrm{mM}$ EDTA, 0.05\% v/v Surfactant P20

547 (HBS-EP+). All measurements were conducted in HBS-EP+ buffer with a flow rate of 30 
$\mu \mathrm{L} /$ minute. The affinity of antibody was analyzed with BIAcore T200 Evaluation software 3.1. A

6. Spike S1 (Omicron): A67V, del69-70, T95I, G142D, del143-145, N211D, del212, G339D, S371L, S373P, S375F, K417N, N440K, G446S, S477N, T478K, E484A, Q493R, G496S, Q498R, N501Y, Y505H, T457K, D614G, H665Y, N679K, P681H, N764K, D796Y, N856K, Q954H, N969K, L981F

561 intravenous injection of 10A3YQYK, $100 \mu \mathrm{L}$ of antibody diluted in $1 \mathrm{X}$ formulation buffer $\mathrm{C}$ was

562 administered retro-orbitally to anesthetized animals. For intranasal injections, antibody was

563 diluted in $1 \mathrm{X}$ formulation buffer $\mathrm{C}$ and administered by inhalation into the nose of anesthetized

564 animals in a total volume of $20-25 \mu \mathrm{L}$ using a pipette tip. Organs, blood, and lung lavage

565 samples were collected 24 hours post-antibody administration. Blood was collected by retro-

566 orbital bleeding and then transferred to Microvette 200 Z-Gel tubes (Cat no\# 20.1291, lot\#

5678071211, SARSTEDT). Tubes were then centrifuged at $10,000 \mathrm{~g}$ for 5 minutes at room 
temperature. Serum was transferred into $1.5 \mathrm{~mL}$ tubes and stored at $-80^{\circ} \mathrm{C}$. Lung lavage samples were collected following insertion of a $20 \mathrm{G} \times 1$-inch catheter (Angiocath Autoguard, Ref\# 381702, lot\# 6063946, Becton Dickinson) into the trachea. A volume of $0.8 \mathrm{~mL}$ of PBS was drawn into a syringe, placed into the open end of the catheter, and slowly injected and aspirated 4 times. The syringe was removed from the catheter, and the recovered lavage fluid was transferred into $1.5 \mathrm{~mL}$ tubes and kept on ice. Lavage samples were centrifuged at $800 \mathrm{~g}$ for $10 \mathrm{~min}$ at $4{ }^{\circ} \mathrm{C}$. Supernatants were collected, transferred to fresh $1.5 \mathrm{~mL}$ tubes, and stored at $-80^{\circ} \mathrm{C}$. Total spleen, total large intestine, total lungs and 200 to $250 \mathrm{mg}$ of small intestine were suspended in $300 \mu \mathrm{L}$ of PBS in pre-filled $2.0 \mathrm{~mL}$ tubes containing zirconium beads (cat 15540945, Spectrum). Tubes were processed in a BeadBug-6 homogenizer at a speed setting of 3,000 and a 30 second cycle time for four cycles with a 30-second break after each cycle. Tissue homogenates were centrifuged at $15,000 \mathrm{rpm}$ for 20 minutes at $4{ }^{\circ} \mathrm{C}$. Homogenate supernatants were then transferred into $1.5 \mathrm{~mL}$ tubes and stored at $-80{ }^{\circ} \mathrm{C}$. STI-9167 antibody levels in each sample were quantified using the antibody detection ELISA method. Statistical significance was determined using the Welch's t-test. This study was reviewed and accepted by the animal study review committee (SRC) and conducted in accordance with IACUC guidelines.

Pharmacokinetic Study

Female CD-1-IGS (strain code \#022) were obtained from Charles River Laboratories at 6-8 weeks of age. STI-9167 was dissolved in intranasal formulation buffer C was administered as previously described for the IN biodistribution study. Lungs and blood were collected from 6 mice at each of the following timepoints: $10 \mathrm{~min}, 1.5 \mathrm{~h}, 6 \mathrm{~h}, 24 \mathrm{~h}, 72 \mathrm{~h}, 96 \mathrm{~h}, 168 \mathrm{~h}, 240 \mathrm{~h}$, and 336 h. Serum and lung tissue samples were collected as described for the biodistribution study. 
STI-9167 antibody levels in each sample were quantified using the antibody detection ELISA method. Pharmacokinetic analysis of the collected ELISA data was performed with the Phoenix WiNnonlin suite of software (version 6.4, Certara) using a non-compartmental approach consistent with an IN-bolus route of administration. Statistical significance was determined using the Welch's t-test. This study was reviewed and accepted by the animal study review committee (SRC) and conducted in accordance with IACUC guidelines.

khACE2 mouse model of COVID-19 infection

K18-hACE2 transgenic mice were purchased from Jackson laboratory and maintained in pathogen-free conditions and handling conforms to the requirements of the National Institutes of Health and the Scripps Research Institute Animal Research Committee. 8-12 weeks old mice were infected intranasally with 10,000 PFU of SARS-COV-2 in total volume $50 \mu \mathrm{L}$ different concentration of $A B$ were injected intravenously $1 \mathrm{~h}$ post infection or by intranasal instillation $12 \mathrm{~h}$ post infection.

\section{Determination of infectious virus titer in the lung}

On day 4 post-infection, animals were euthanized, lung tissue samples were collected from each animal, and the left lobe of each collected lung was placed into a pre-labeled

606 microcentrifuge tube containing 3-5 beads $2.3 \mathrm{~mm}$ diameter Zirconia/silica beads (Fischer).

607 Lung samples were homogenized with DMEM + 5\% FBS in a Tissuelyser 1 min $25 \sec ^{55}$. 
611 methylcellulose [Sigma] and culture media) is added to each well and incubation commenced

612 for 3 days at $37^{\circ} \mathrm{C}$. Plaque staining was performed using Crystal Violet as mentioned above.

613 Virus titers in lungs were compared with the isotype control mAb-treated group using a

614 Student's t-test.

615 Plaque reduction neutralizing assay

616 VeroE6 cells were plated at $18.0 \mathrm{E}+03$ cells/well in a flat bottom 96-well plate in a volume of 200

$617 \mu \mathrm{L} /$ well. After $24 \mathrm{~h}$, a serial dilution of $\mathrm{ABs}$ is prepared in a $100 \mu \mathrm{L} /$ well at twice the final

618 concentration desired and live virus was added at 1,000 PFU/100 $\mu \mathrm{L}$ of SARS-COV-2 and

619 subsequently incubated for $1 \mathrm{~h}$ at $37^{\circ} \mathrm{C}$ in a total volume of $200 \mu \mathrm{L} /$ well. Cell culture media was

620 removed from cells and sera/virus premix was added to VeroE6 cells at $100 \mu \mathrm{L} /$ well and

621 incubated for $1 \mathrm{~h}$ at $37^{\circ} \mathrm{C}$. After incubation, $100 \mu \mathrm{L}$ of "overlay" (1:1 of $2 \%$ methylcellulose

622 (Sigma) and culture media) is added to each well and incubation commenced for $3 \mathrm{~d}$ at $37^{\circ} \mathrm{C}$.

623 Plaque staining using Crystal Violet (Sigma) was performed upon 30 min of fixing the cells with

624 4\% paraformaldehyde (Sigma) diluted in PBS. Plaques were assessed using a light microscope

625 (Keyence). 


\section{ACKNOWLEDGEMENTS}

628 This work was partly supported by CRIPT (Center for Research on Influenza Pathogenesis and

629 Transmission), an NIAID funded Center of Excellence for Influenza research and Response

630 (CEIRR, contract \#75N93021R00014 to AGS, MS, FK), by DARPA grant HR0011-19-2-0020 (to

631 AGS) and by NCl Seronet grant U54CA260560 (to AGS, MS, FK). We thank R. Albrecht for

632 support with the BSL-3 facility and procedures at the Icahn School of Medicine at Mount Sinai,

633 NY. S.Y. received funding from Swiss National Foundation (SNF) Postdoc Mobility fellowship

634 (P400PB_199292). M.S. laboratory is supported by NIH grant R01DK130425.

\section{DISCLOSURES}

636 The A.G.-S. laboratory has received research support from Pfizer, Senhwa Biosciences, Kenall

637 Manufacturing, Avimex, Johnson \& Johnson, Dynavax, 7Hills Pharma, Pharmamar, ImmunityBio,

638 Accurius, Nanocomposix, Hexamer, N-fold LLC, Model Medicines, Atea Pharma and Merck,

639 outside of the reported work. A.G.-S. has consulting agreements for the following companies

640 involving cash and/or stock: Vivaldi Biosciences, Contrafect, 7Hills Pharma, Avimex, Vaxalto,

641 Pagoda, Accurius, Esperovax, Farmak, Applied Biological Laboratories, Pharmamar, Paratus,

642 CureLab Oncology, CureLab Veterinary and Pfizer, outside of the reported work. A.G.-S. is

643 inventor on patents and patent applications on the use of antivirals and vaccines for the

644 treatment and prevention of virus infections and cancer, owned by the Icahn School of

645 Medicine at Mount Sinai, New York, outside of the reported work. 
bioRxiv preprint doi: https://doi.org/10.1101/2022.01.19.476998; this version posted January 20, 2022. The copyright holder for this preprint (which was not certified by peer review) is the author/funder. All rights reserved. No reuse allowed without permission.

648 
FIGURE LEGENDS

650 Figure 1. Rapid Discovery of Neutralizing Antibodies. (A) Harbour H2L2 Mice ${ }^{\circledR}(M-1,-2,-3,-4)$

651 were immunized and boosted 2X with SARS-CoV-2 RBD (Wuhan strain) and (B) sera (1:100,

652 1:500, and 1:2,500) from these mice were analyzed by flow cytometry from Expi293F

653 untransfected or transfected with SARS-Cov-2 spike. As a control, serum from a non-immunized

654 mouse was used. (C) A primary screen based on the anti-RBD clones from mouse 4(M-4) was

655 performed using flow-cytometry using HEK-293 cells transfected with spike protein and RBD

656 ELISA. Upon flow cytometry analysis, the mean fluorescence intensity (MFI) was determined for

657 each clone. The RBD-ELISA represents binding of the clones to RBD as measured by absorbance.

658 Both the flow cytometry and ELISA data are represented as heat maps. The secondary assay for

659 the binding clones was a neutralization assay using VSV-spike ${ }^{\text {CoV-2 }}$ followed by a determination

660 of IC50 (pM) for clones with > 50\% neutralization activity. (D) The clones with IC50 values $<500$

$661 \mathrm{pM}$ were sequenced and $\mathrm{mAb}$ clones were identified by specific $\mathrm{V}(\mathrm{D}) \mathrm{J}$ gene-segment

662 combinations and junction (CDR3) characteristics, which allowed them to be grouped into

663 different clonal families (Family “A-G"). 
665 Figure 2. Binding and neutralization of candidate antibody. (A) Affinity measurements of STI-

6669167 for Spike S1 binding domain from the following isolates and VOCs: USA/WA-1/2020(WA-1)

667 isolate, Delta, and Omicron. The antibody affinities were measured using SPR on a BIAcore T200

668 instrument using a 1:1 binding model. (B) Spike protein derived from WA-1, Delta, Omicron,

669 and Omicron + R346K SARS-CoV-2 isolates were independently expressed on the surface of HEK

670293 cells. Serially-diluted STI-9167 was assayed for Spike protein binding by flow cytometry. To

671 quantify antibody binding, mean fluorescent intensity was measured for each dilution tested

672 and the $\mathrm{EC}_{50}$ value was calculated for each $\mathrm{nAb}$. Representative replicate experiments are

673 shown. (C) Spike-pseudotyped VSV neutralization. Antibody neutralization of the indicated

674 spike variant pseudotyped VSVs was performed as described in the methods. The curves

675 represent the average of three independent experiments, with error bars representing one

676 standard deviation. IC50 values for each pseudotype/antibody combination are indicated on

677 the right. (D) PRNT assay using STI-9167 with indicated SARS-COV-2 variants were performed

678 as described in the methods, presenting percent neutralization and the calculated IC50 values

679 indicated on the right. 
681 Figure 3. Pharmacokinetic and bioavailability of Neutralizing Antibody. Biodistribution:

682 Concentration of STI-9167 in serum and lung lavage or lysates of spleens, lungs, small

683 intestines, and large intestines collected from female CD-1 mice administered STI-9167 (A) IV

684 at doses of $0.5 \mathrm{mg} / \mathrm{kg}(\bullet), 0.05 \mathrm{mg} / \mathrm{kg}(\bullet)$, or $0.005 \mathrm{mg} / \mathrm{kg}(\odot)$ or $(\mathrm{B})$ IN at doses of $0.5 \mathrm{mg} / \mathrm{kg}$

$685(\bullet), 0.05 \mathrm{mg} / \mathrm{kg}(\bullet)$, and $0.005 \mathrm{mg} / \mathrm{kg}(\odot)$ at 24 hours post-administration as compared to

686 samples collected from untreated mice. Values represent mean \pm SEM ( $n=3-4$ animals no

687 treatment group, $\mathrm{n}=5$ in treatment groups). Significant differences are denoted by *, $\mathrm{P}<0.05$;

$688 * *, \mathrm{P}<0.01 ; * * *, \mathrm{P}<0.001, * * * *, \mathrm{P}<0.0001$. Pharmacokinetics: Concentration of STI-9167 (C)

689 in lungs and isolated serum collected from female CD-1 mice administered STI-9167 intranasally

690 (IN) at a dose of $5 \mathrm{mg} / \mathrm{kg}$. Samples from treated mice were collected at the indicated timepoint

691 post-administration; antibodies concentrations were quantified by ELISA and compared to

692 samples collected from untreated mice. Values represent mean \pm SD ( $n=3-6$ animals no

693 treatment group, $\mathrm{n}=6$ per time point in treatment groups).

694

695 
Figure 4. Efficacy of Intranasal (IN) delivery of STI-9167 Neutralizing Antibody in the K18hACE2 murine model of COVID-19.

(A) K18-hACE2 transgenic mice were infected with 10,000 PFU of WA-1, Delta or Omicron SARSintravenously $1 \mathrm{~h}$ post infection. (B) Body weight change of mice was measured daily $(n=5)$. (C) SARS-CoV-2 viral titers were measured in lung day 4 post infection $(n=5)$. (D) K18-hACE2 transgenic mice were infected with 10,000 PFU SARS-CoV-2 WA1, Delta, or Omicron strains and treated with indicated concentration of Isotype or STI-9167 intranasally $12 \mathrm{~h}$ post infection.(E)

704 Body weight change of mice was measured daily $(n=5)$. (F) SARS-CoV-2 viral titers were measured in lung day 4 post infection $(\mathrm{n}=5)$ n.s. not significant, $\mathrm{P}^{*}<0.05, \mathrm{P}^{* *}<0.01, \mathrm{P}^{* * *}<$ 
712 Table 1. Binding and Neutralization of various Neutralizing Antibodies to SARS-CoV-2 and

713 select VOC. (A) Omicron spike S1 binding affinity to indicated nAbs. (B) Spike protein from

714 selected VOCs expressed on HEK 293 cells binding to presented nAbs expressed as EC50

$715 \quad(\mu \mathrm{g} / \mathrm{mL})$. (C) Spike-pseudotyped VSV neutralization of indicated nAbs. (D) Live virus

716 neutralization on Vero and VERO-ACE2 cells for WA-1 and Omicron virus, using indicated nAbs. 


\section{Supplemental Figure 1. Binding and neutralization of candidate antibody to VoCs.}

718 (A) Affinity measurements of STI-9167 for Spike S1 binding domain from the following isolates 719 and VOCs: USA/WA-1/2020(WA-1) isolate, Alpha, Beta, and Gamma. The antibody affinities were 720 measured using SPR on a BIAcore T200 instrument using a 1:1 binding model. Graphs are

721 representative of triplicate data and table data presented as mean \pm SD. (B) Spike protein derived 722 from Alpha, Beta, Gamma, Delta Plus, and Lambda SARS-CoV-2 isolates were independently 723 expressed on the surface of HEK 293 cells. Serially-diluted STI-9167 was assayed for Spike protein 724 binding by flow cytometry. To quantify antibody binding, mean fluorescent intensity was 725 measured for each dilution tested and the $\mathrm{EC}_{50}$ value was calculated for each nAb. (C) Spike726 pseudotyped VSV neutralization. Antibody neutralization of the indicated spike variant 727 pseudotyped VSVs was performed as described in the methods. The curves represent the average 728 of three independent experiments, with error bars representing one standard deviation. IC50 729 values for each pseudotype/antibody combination are indicated on the right. (D) PRNT assay 730 using STI-9167 with indicated SARS-COV-2 variants were performed as described in the methods.

Supplemental Figure 2. Efficacy of Intranasal (IN) delivery of STI-9167 Neutralizing Antibody in the 733 K18-hACE2 murine model of COVID-19 VoCs.

734 (A) A schematic of experimental model, K18-hACE2 transgenic mice were infected with 10000 PFU of indicated variants of SARS-CoV-2 treated with indicated concentration of AB (STI-9167) intravenously 1 hour post infection. (B) Body weight change of mice was measured daily $(n=5)$. 
740 Supplemental Figure 3. Plaque Reduction Neutralization Fluorescent staining on Vero-ACE2

741 cells. PRNT assay using STI-9167 and various neutralizing antibodies with SARS-COV-2 WA-1 or

742 Omicron were performed as described in the methods on Vero-ACE2-expressing cells and

743 visualized. 


\section{References}

756

757

758

759

760

761

762

763

764

765

766

767

768

769

770

771

772

773

774

775

776

777

778

779

780

781

782

783

784

785

786

787

788

789

790

791

792

793

794

795

796

797

798

799

800
1 Zhu, N. et al. A Novel Coronavirus from Patients with Pneumonia in China, 2019. N Engl J Med 382, 727-733, doi:10.1056/NEJMoa2001017 (2020).

2 Ahn, D. G. et al. Current Status of Epidemiology, Diagnosis, Therapeutics, and Vaccines for Novel Coronavirus Disease 2019 (COVID-19). J Microbiol Biotechnol 30, 313-324, doi:10.4014/jmb.2003.03011 (2020).

3 Amanat, F. \& Krammer, F. SARS-CoV-2 Vaccines: Status Report. Immunity 52, 583-589, doi:10.1016/j.immuni.2020.03.007 (2020).

4 Mascola, J. R., Graham, B. S. \& Fauci, A. S. SARS-CoV-2 Viral Variants-Tackling a Moving Target. JAMA 325, 1261-1262, doi:10.1001/jama.2021.2088 (2021).

$5 \quad H o f f m a n n, M$. et al. The Omicron variant is highly resistant against antibody-mediated neutralization: Implications for control of the COVID-19 pandemic. Cell, doi:10.1016/j.cell.2021.12.032 (2021).

6 Bedford, J. et al. COVID-19: towards controlling of a pandemic. Lancet 395, 1015-1018, doi:10.1073/pnas.88.18.7978 (2020).

$7 \quad$ Uriu, K. et al. Neutralization of the SARS-CoV-2 Mu Variant by Convalescent and Vaccine Serum. New England Journal of Medicine 385, 2397-2399, doi:10.1056/NEJMc2114706 (2021).

8 Cameroni, E. et al. Broadly neutralizing antibodies overcome SARS-CoV-2 Omicron antigenic shift. bioRxiv, 2021.2012.2012.472269, doi:10.1101/2021.12.12.472269 (2021).

9 Aggarwal, A. et al. SARS-CoV-2 Omicron: evasion of potent humoral responses and resistance to clinical immunotherapeutics relative to viral variants of concern. medRxiv, 2021.2012.2014.21267772, doi:10.1101/2021.12.14.21267772 (2021).

10 Planas, D. et al. Considerable escape of SARS-CoV-2 variant Omicron to antibody neutralization. bioRxiv, 2021.2012.2014.472630, doi:10.1101/2021.12.14.472630 (2021).

11 Cao, Y. et al. Omicron escapes the majority of existing SARS-CoV-2 neutralizing antibodies. bioRxiv, 2021.2012.2007.470392, doi:10.1101/2021.12.07.470392 (2021).

12 Liu, L. et al. Striking Antibody Evasion Manifested by the Omicron Variant of SARS-CoV-2. bioRxiv, 2021.2012.2014.472719, doi:10.1101/2021.12.14.472719 (2021).

13 VanBlargan, L. A. et al. An infectious SARS-CoV-2 B.1.1.529 Omicron virus escapes neutralization by several therapeutic monoclonal antibodies. bioRxiv, 2021.2012.2015.472828, doi:10.1101/2021.12.15.472828 (2021).

14 Cao, X. et al. Discovery and Development of Human SARS-CoV-2 Neutralizing Antibodies using an Unbiased Phage Display Library Approach. bioRxiv, 2020.2009.2027.316174, doi:10.1101/2020.09.27.316174 (2020).

15 Elbe, S. \& Buckland-Merrett, G. Data, disease and diplomacy: GISAID's innovative contribution to global health. Global Challenges 1, 33-46, doi:https://doi.org/10.1002/gch2.1018 (2017).

16 Julia L. Mullen, G. T., Alaa Abdel Latif, Manar Alkuzweny, Marco Cano, Emily Haag, Jerry Zhou, Mark Zeller, Emory Hufbauer, Nate Matteson, Kristian G. Andersen, Chunlei Wu, Andrew I. Su, Karthik Gangavarapu, Laura D. Hughes, and the Center for Viral Systems Biology. (2020).

17 Poh, C. M. et al. Two linear epitopes on the SARS-CoV-2 spike protein that elicit neutralising antibodies in COVID-19 patients. Nat Commun 11, 2806, doi:10.1038/s41467-020-16638-2 (2020).

18 Gupta, A. et al. Early Treatment for Covid-19 with SARS-CoV-2 Neutralizing Antibody Sotrovimab. N Engl J Med 385, 1941-1950, doi:10.1056/NEJMoa2107934 (2021).

19 Pinto, D. et al. Cross-neutralization of SARS-CoV-2 by a human monoclonal SARS-CoV antibody. Nature 583, 290-295, doi:10.1038/s41586-020-2349-y (2020). 


\section{Figure 1}

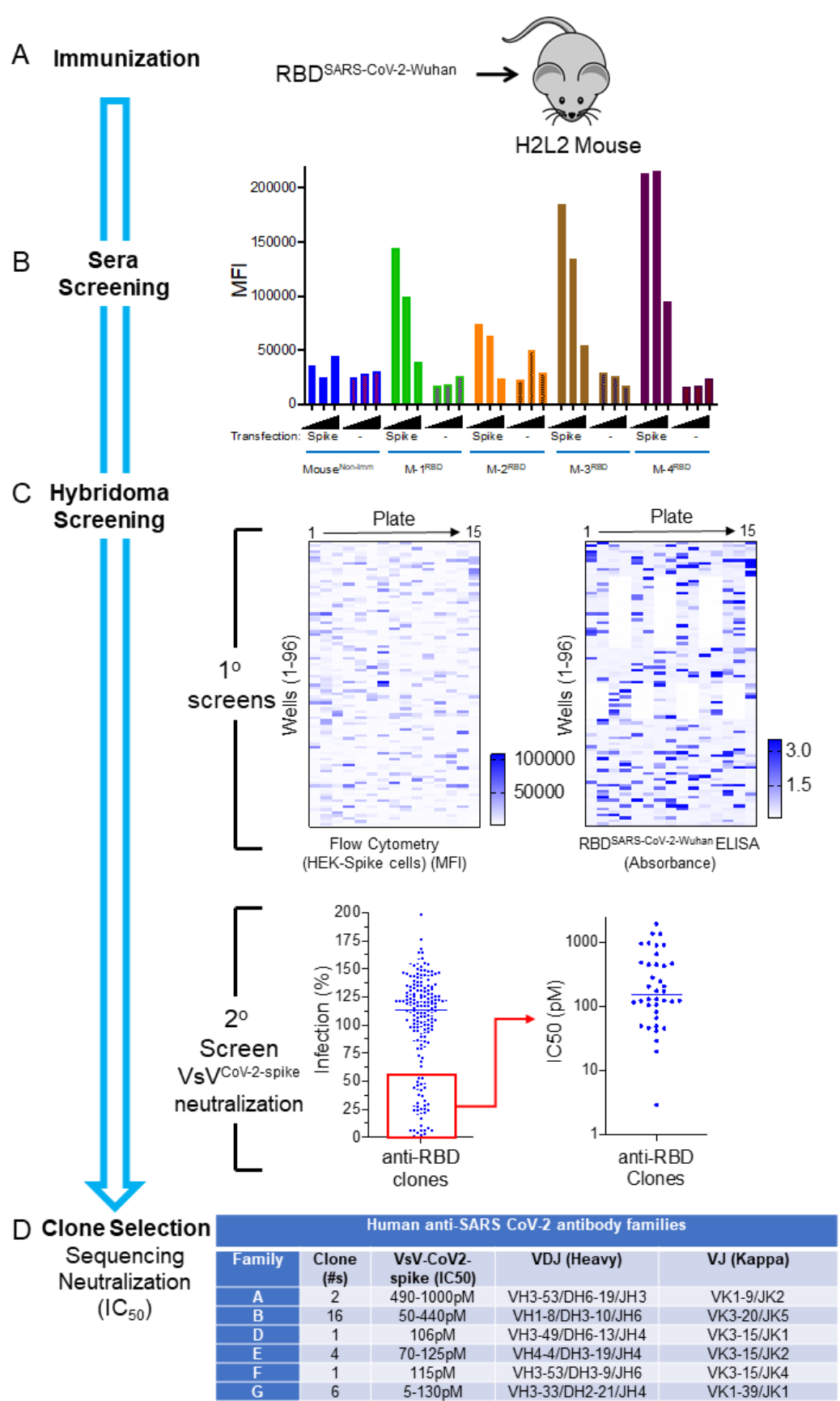




\section{Figure 2}

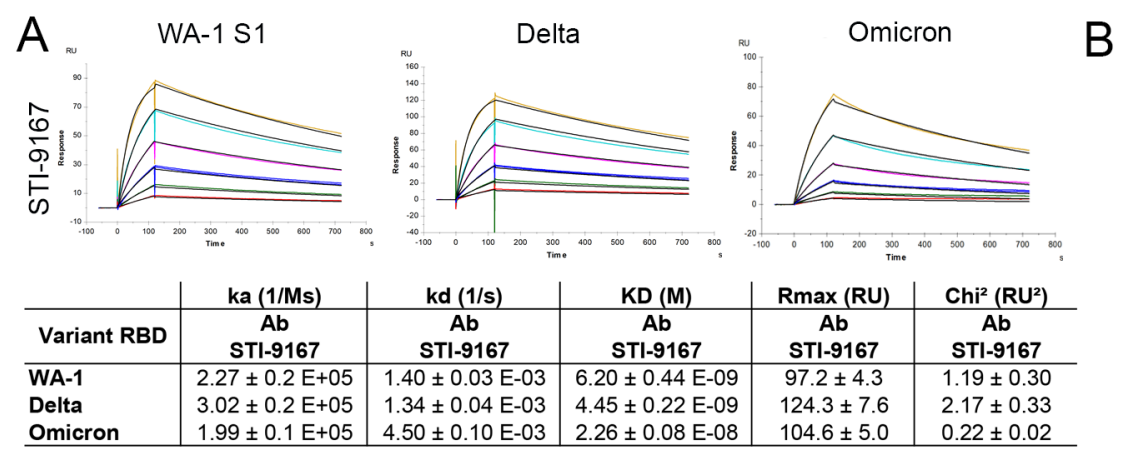

\begin{tabular}{|c|c|}
\hline \multicolumn{2}{|c|}{$\begin{array}{c}\text { cell-expressed spike } \\
\text { binding EC50 ( } \mathrm{gg} / \mathrm{mL})\end{array}$} \\
\hline $\begin{array}{c}\begin{array}{c}\text { Variant } \\
\text { spike }\end{array} \\
\end{array}$ & $\begin{array}{c}A b \\
\text { STI-9167 }\end{array}$ \\
\hline WA-1 & 0.025 \\
\hline Delta & 0.011 \\
\hline Omicron & 0.025 \\
\hline Omicron + & 0.023 \\
\hline
\end{tabular}

\begin{tabular}{l|l|l|l|l|l} 
Delta & $3.02 \pm 0.2 \mathrm{E}+05$ & $1.34 \pm 0.04 \mathrm{E}-03$ & $4.45 \pm 0.22 \mathrm{E}-09$ & $124.3 \pm 7.6$ & $2.17 \pm 0.33$ \\
Omicron & $1.99 \pm 0.1 \mathrm{E}+05$ & $4.50 \pm 0.10 \mathrm{E}-03$ & $2.26 \pm 0.08 \mathrm{E}-08$ & $104.6 \pm 5.0$ & $0.22 \pm 0.02$ \\
\hline
\end{tabular}

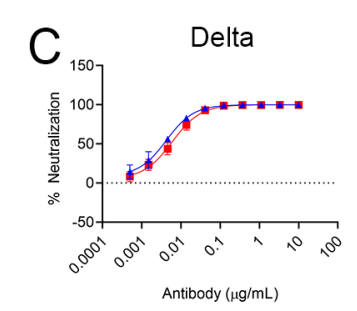

D

$\rightarrow$ STI-9167

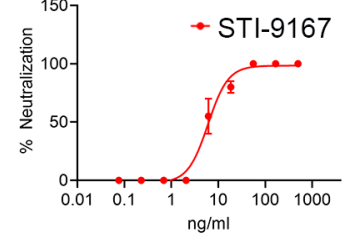

Omicron

Pseudotype neutralization
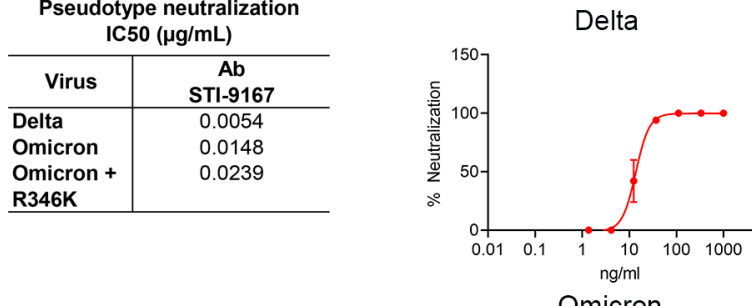

Virus neutralization IC50

Omicron + R346K

R346K
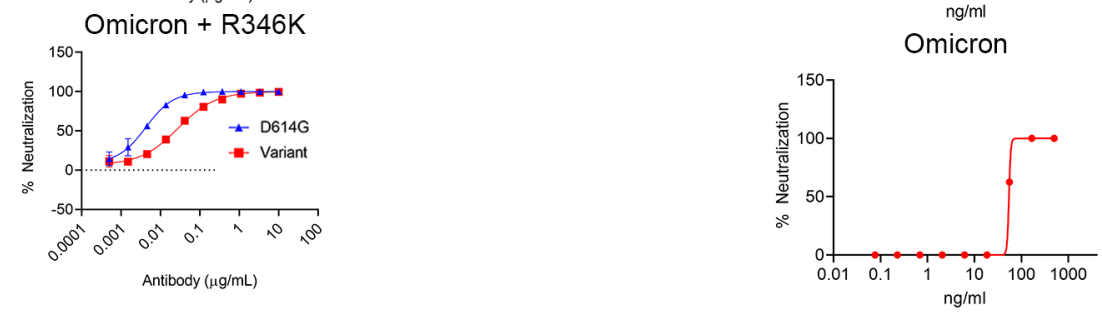

\section{\begin{tabular}{c|c} 
Virus & Ab \\
& STI-9167 \\
\hline
\end{tabular}} \begin{tabular}{l|c|}
\hline WA-1 & 6.041 \\
\hline
\end{tabular} \begin{tabular}{l|l} 
Delta & 13.7 \\
\hline Omicron & 5429 \\
\hline
\end{tabular} Omicron $\quad 54.29$ 
Figure 3
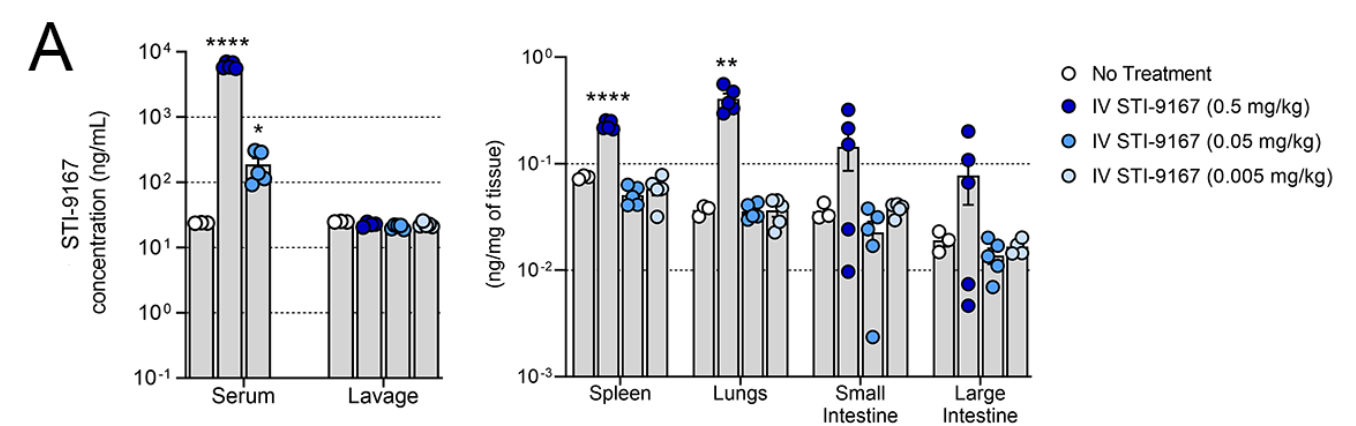

B

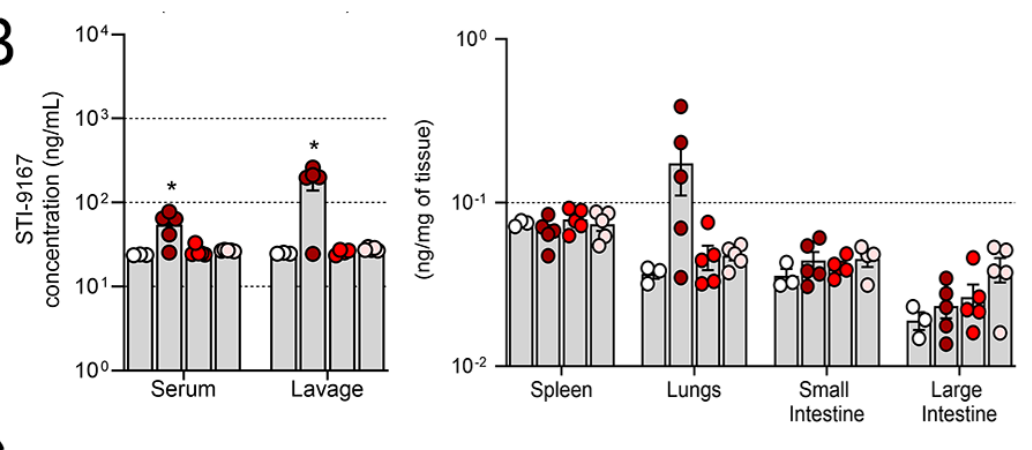

C

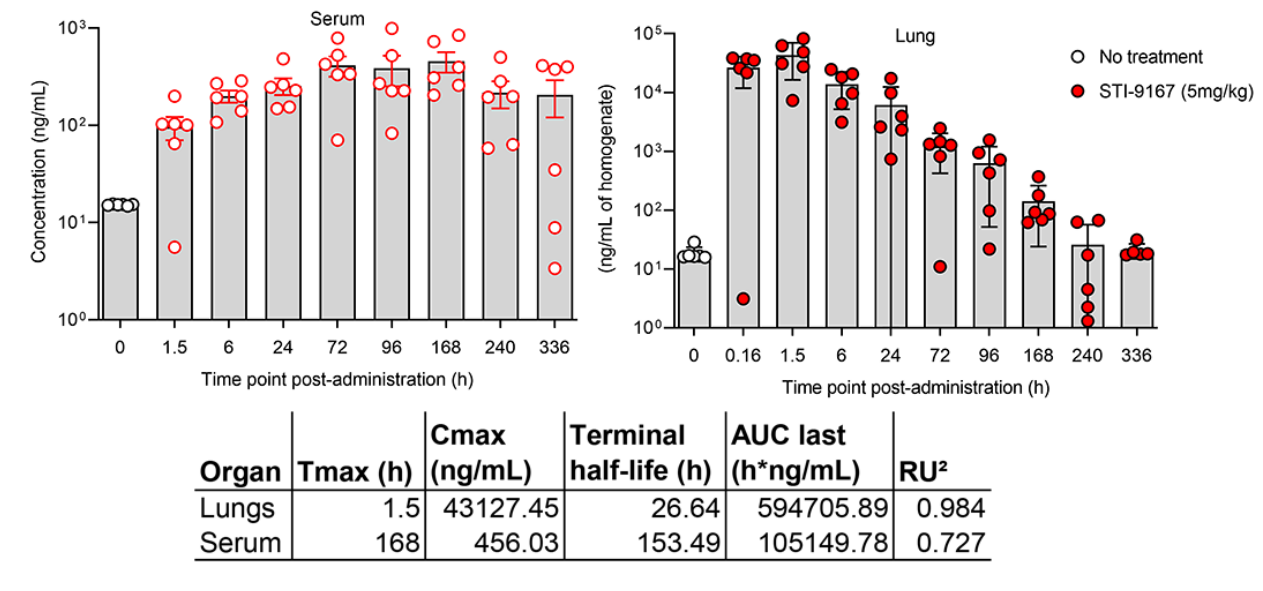




\section{Figure 4}
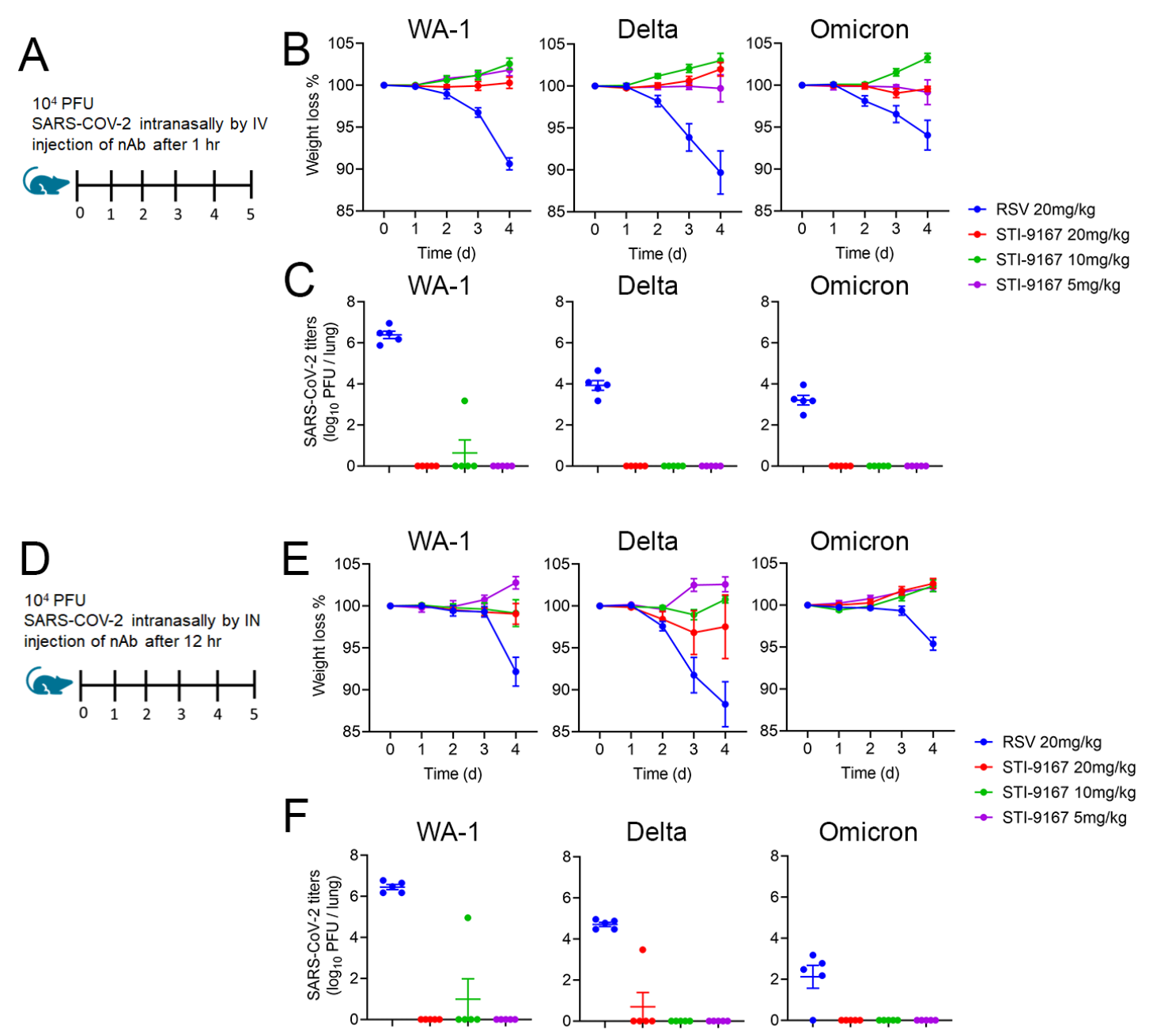


\section{Table 1}

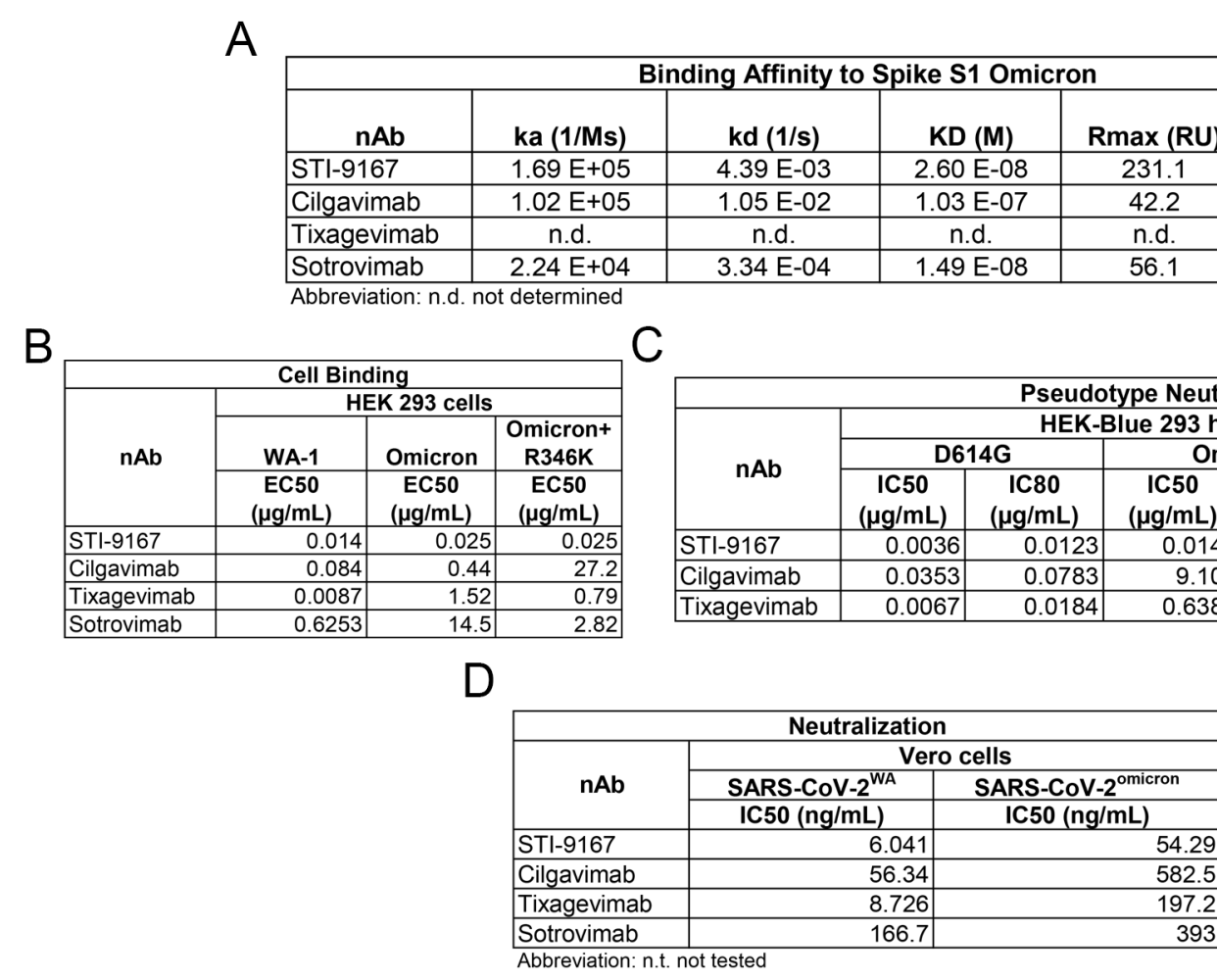




\section{Supplemental Figure 1}

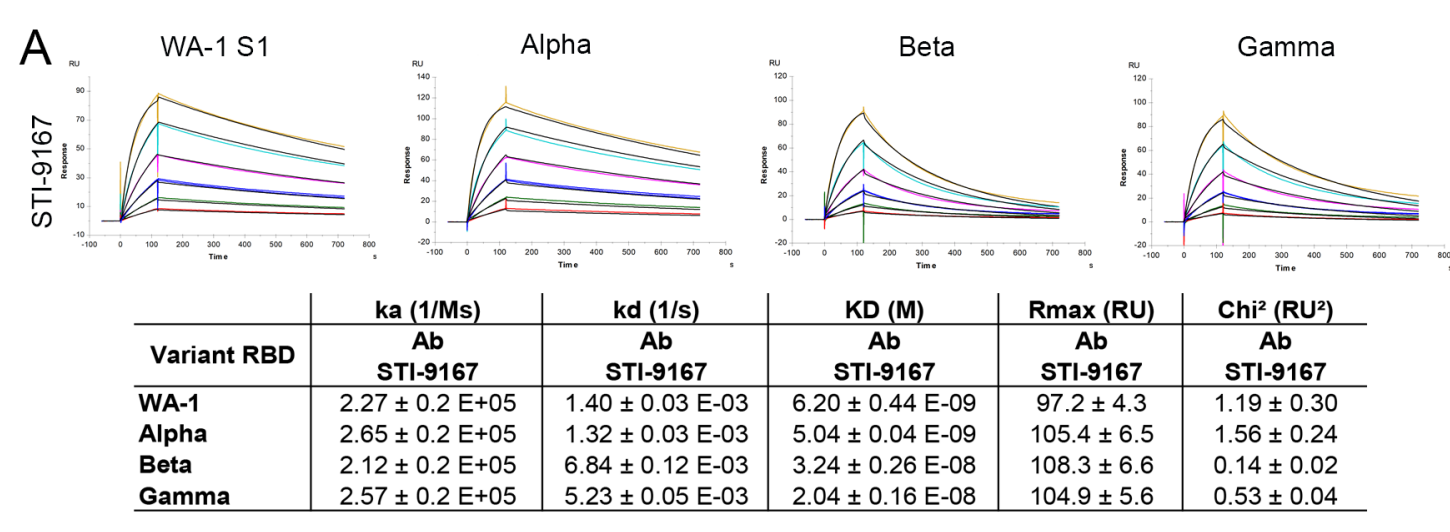

\section{B}

cell-expressed spike binding

$$
\text { EC50 }(\mu \mathrm{g} / \mathrm{mL})
$$

\begin{tabular}{l|c}
\hline Variant spike & $\mathrm{Ab}$ \\
\hline STI-9167 \\
\hline WA-1 & 0.025 \\
Alpha & 0.019 \\
Beta & 0.015 \\
Gamma & 0.014 \\
Delta Plus & 0.043 \\
\hline
\end{tabular}
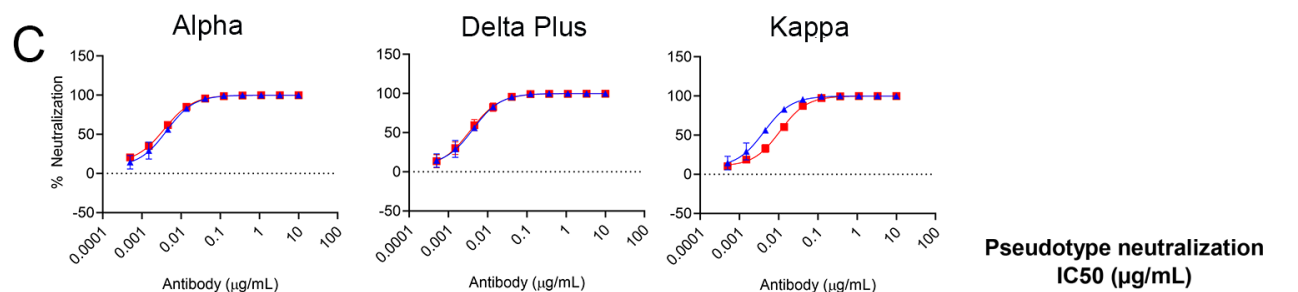

D Alpha
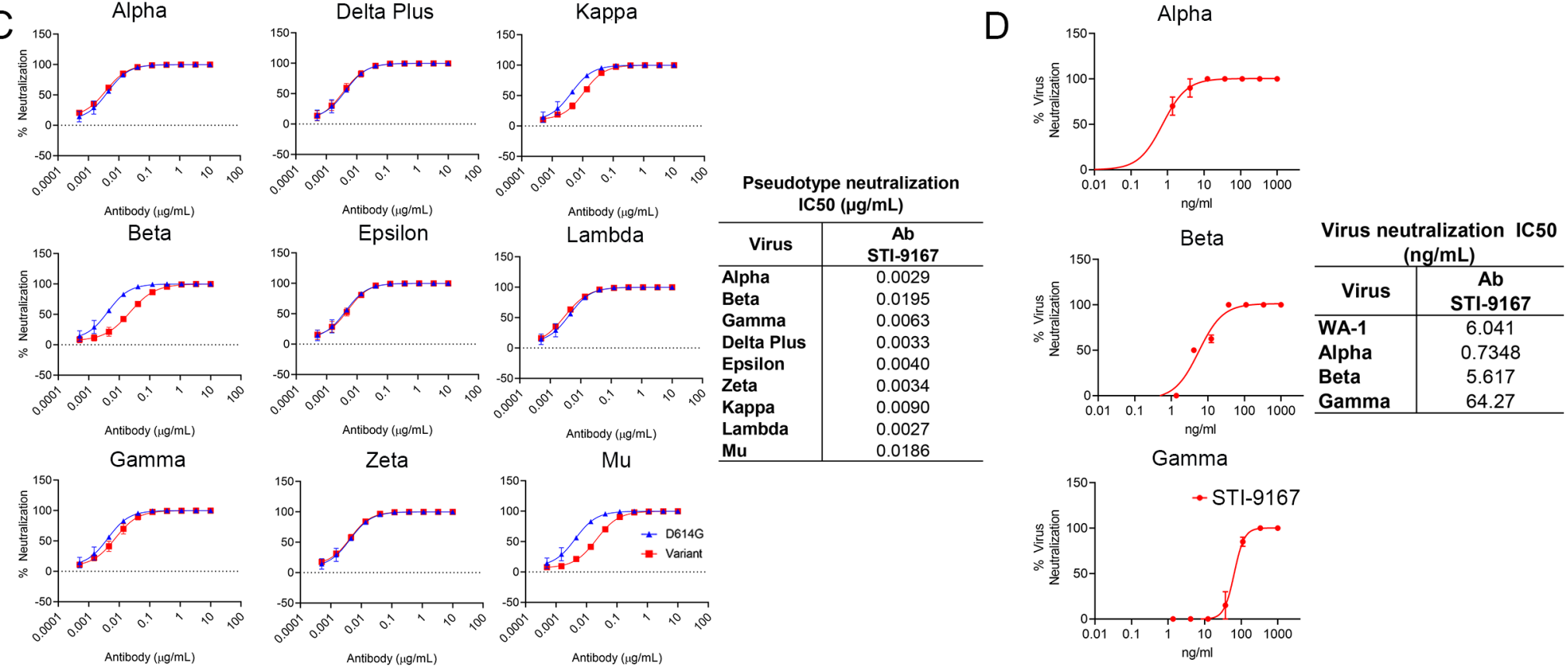
Supplemental Figure 2
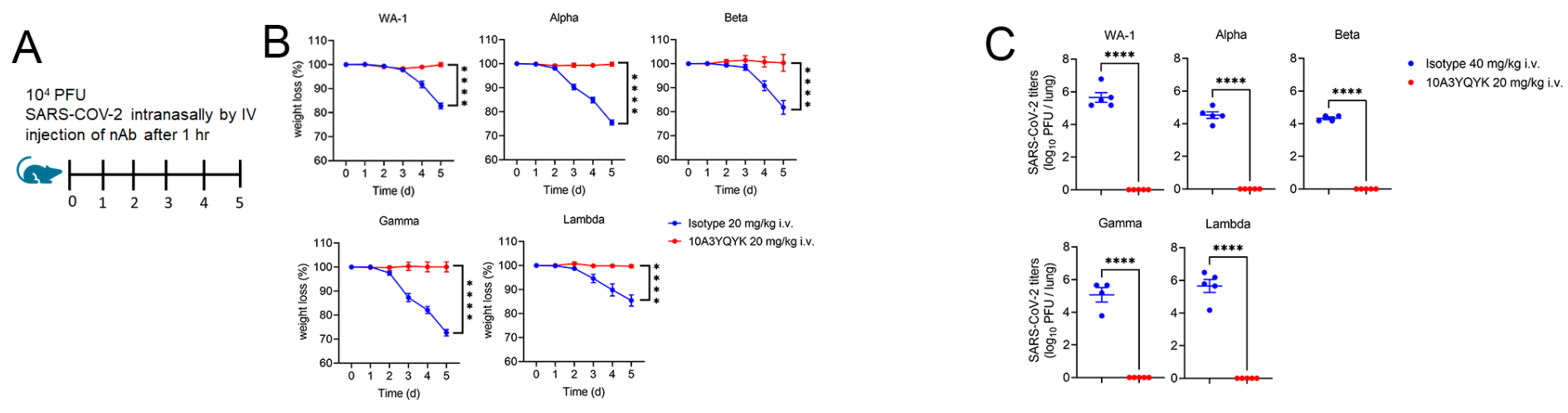
Supplemental Figure 3
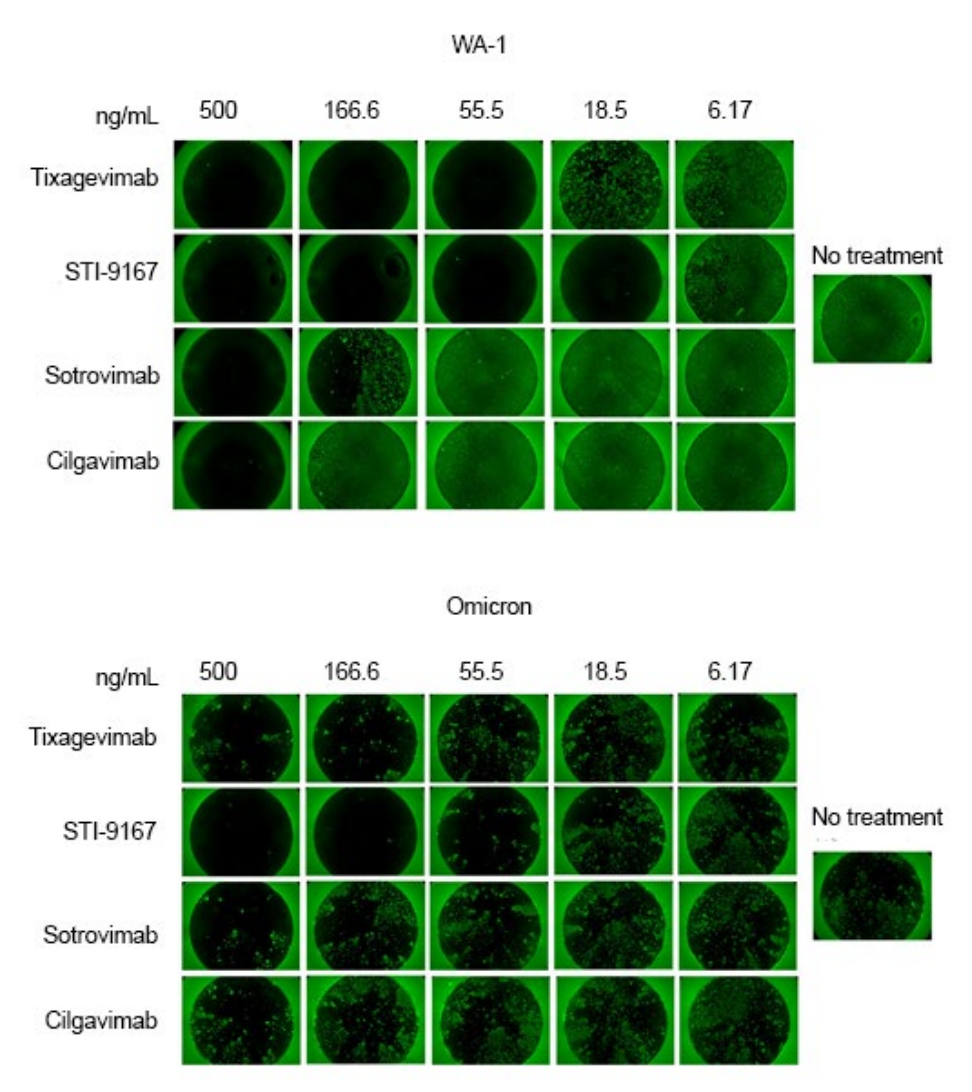
Supplemental Figure 4

Binding Affinity Spike S1 Omicron

STI-9167

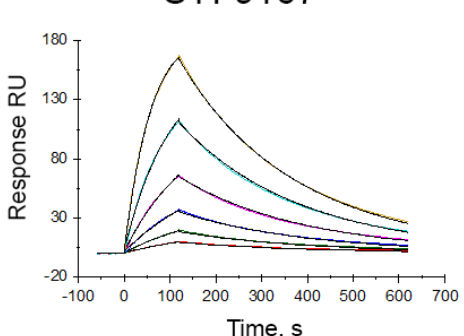

Cilgavimab

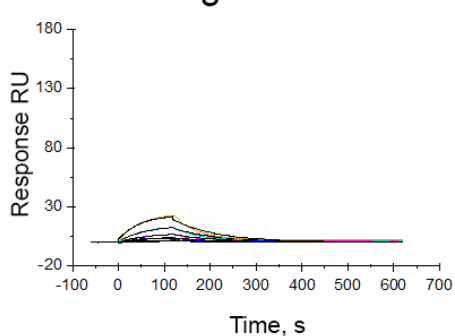

Sotrovimab

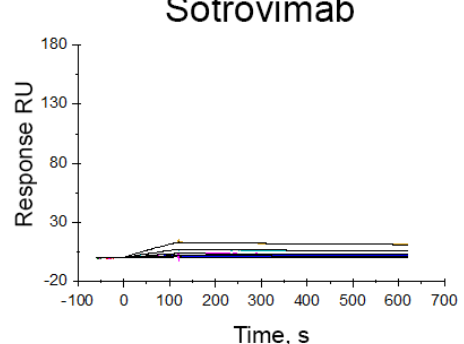

Cilgavimab

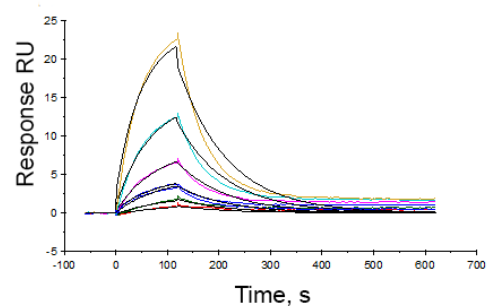

Sotrovimab

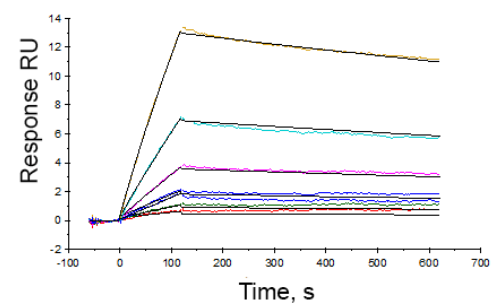


Supplemental Figure 5
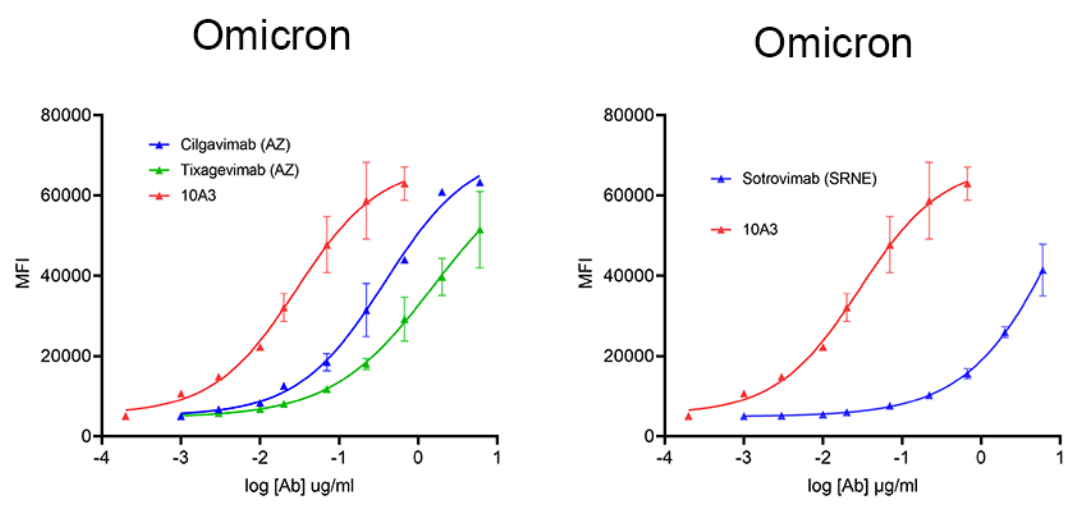